\title{
Copper-promoted Double Oxidative C-H Amination Cascade for the Synthesis of Imidazo[1,5-a]quinolines
}

\author{
Zhong Li ${ }^{\dagger}$, Song-Song Wu ${ }^{\dagger}$, Zai-Gang Luo ${ }^{\dagger}$, Wei-Kang Liu ${ }^{\dagger}$, Cheng-Tao Feng ${ }^{\dagger} *$ and \\ Shi-Tang Ma *
}

${ }^{\dagger}$ School of Chemical Engineering, Anhui University of Science and Technology, Huainan, Anhui 232001, P. R. China

E-mail: fengct2010@163.com

${ }^{\ddagger}$ College of Food and Drug, Anhui Science and Technology University, Fengyang, Anhui 233100, P. R. China

E-mail: nothingchina@126.com

\section{Supporting Information}

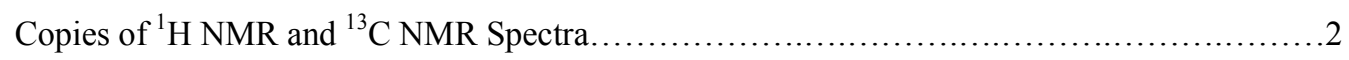




\section{Copies of ${ }^{1} \mathrm{H}$ NMR and ${ }^{13} \mathrm{C}$ NMR Spectra}

1-phenylimidazo[1,5-a]quinoline (3aa).
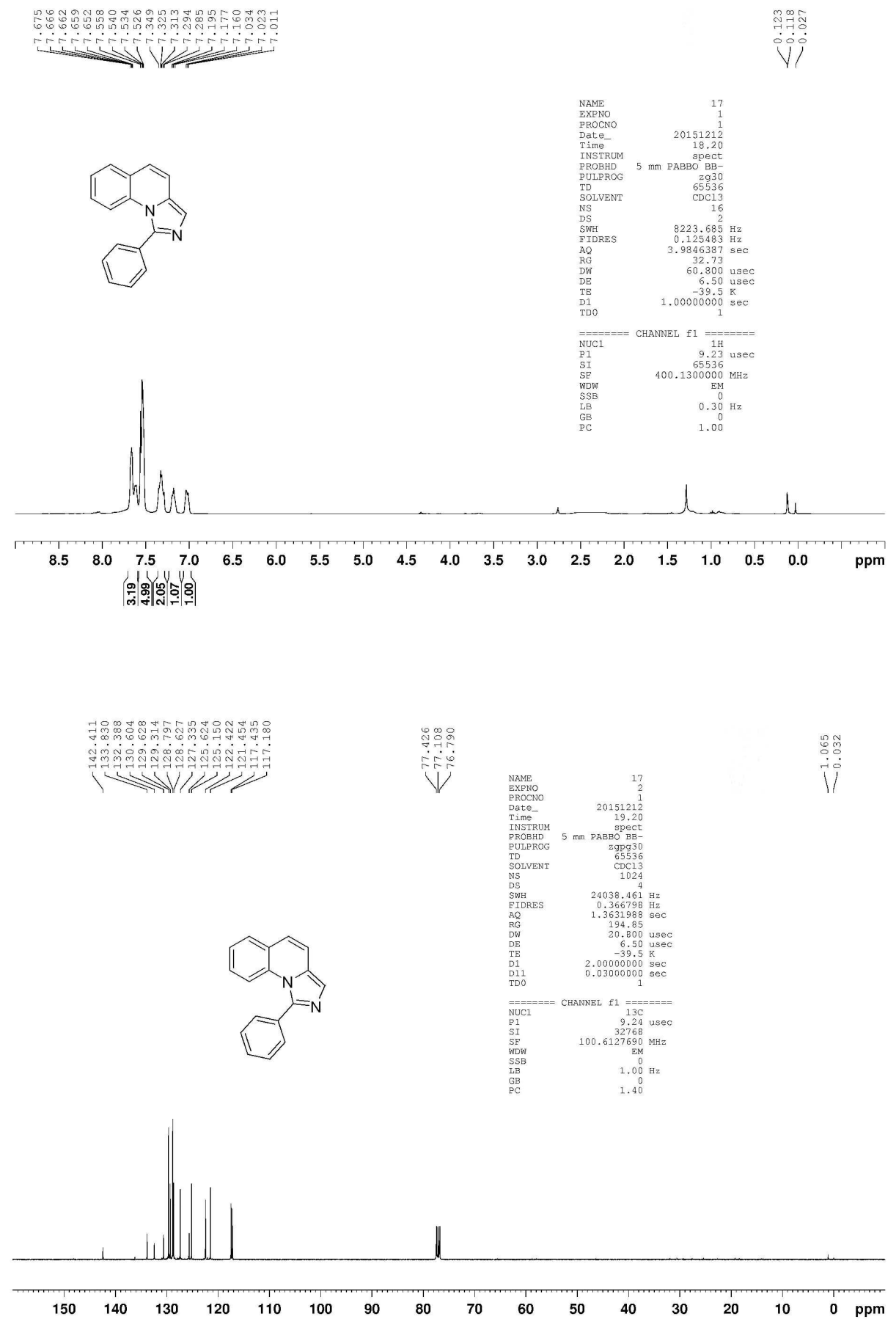
1-(p-tolyl)imidazo[1,5-a]quinoline (3ab).
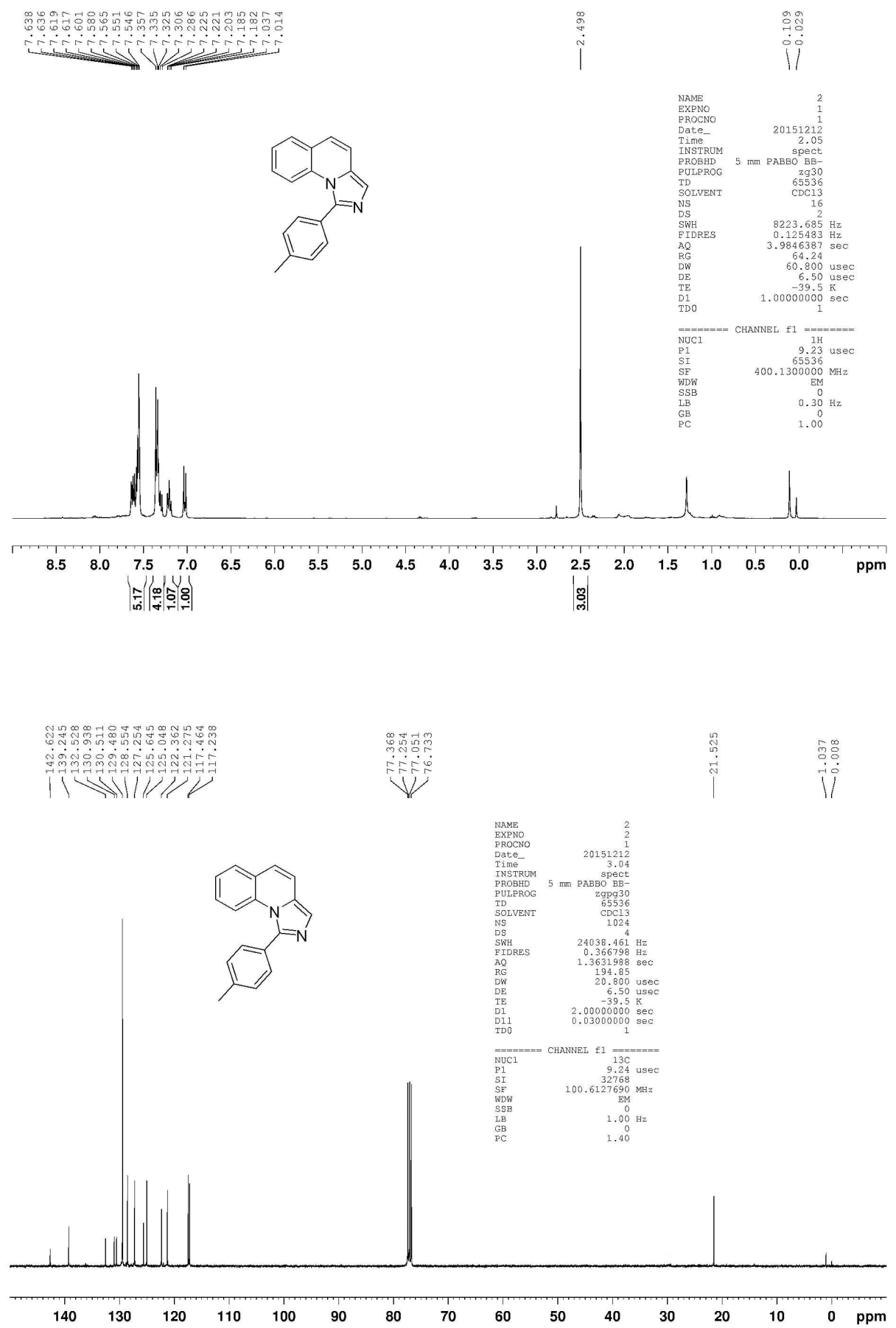
1-(m-tolyl)imidazo[1,5-a]quinoline (3ac).
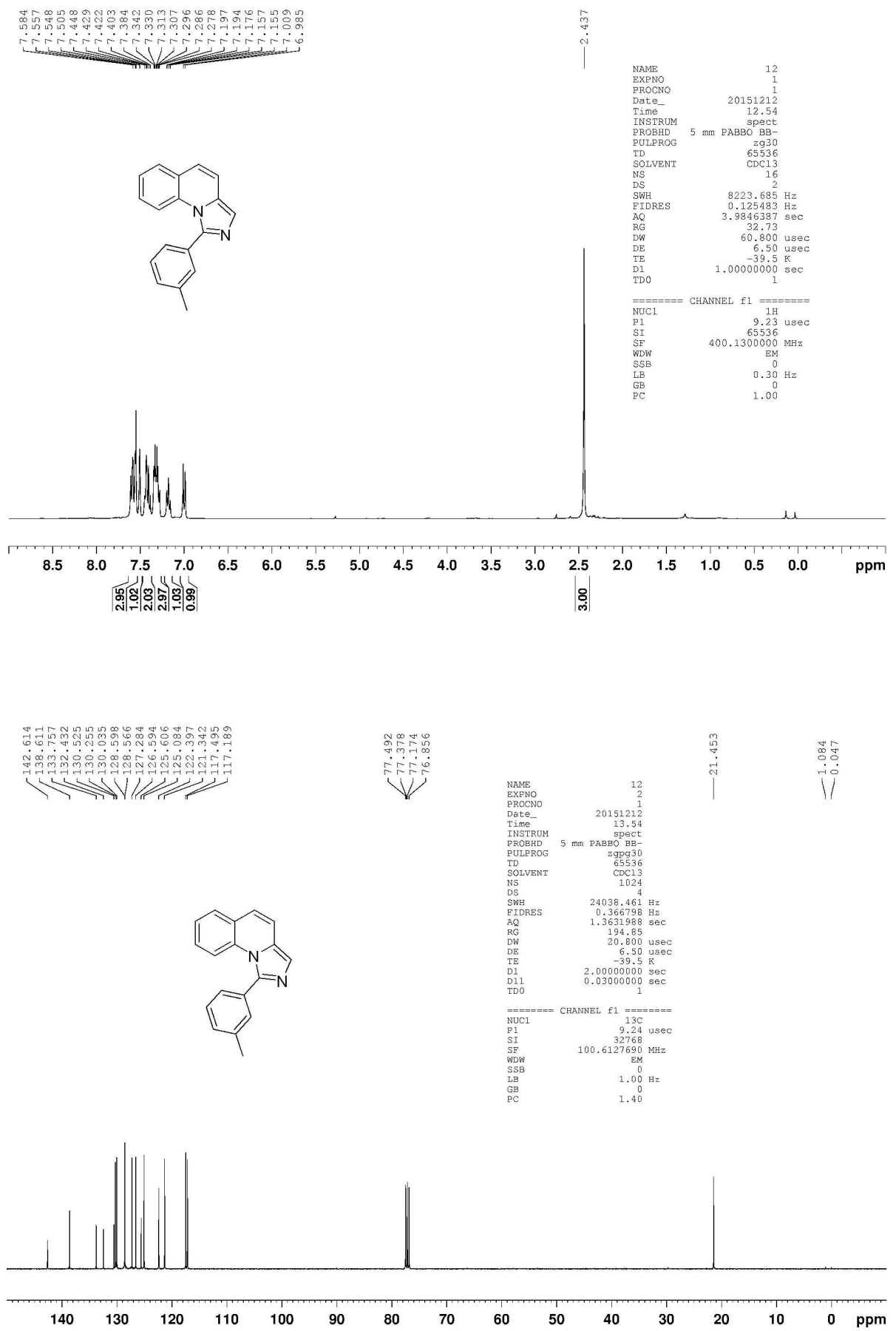
1-(o-tolyl)imidazo[1,5- $a$ ]quinoline (3ad).
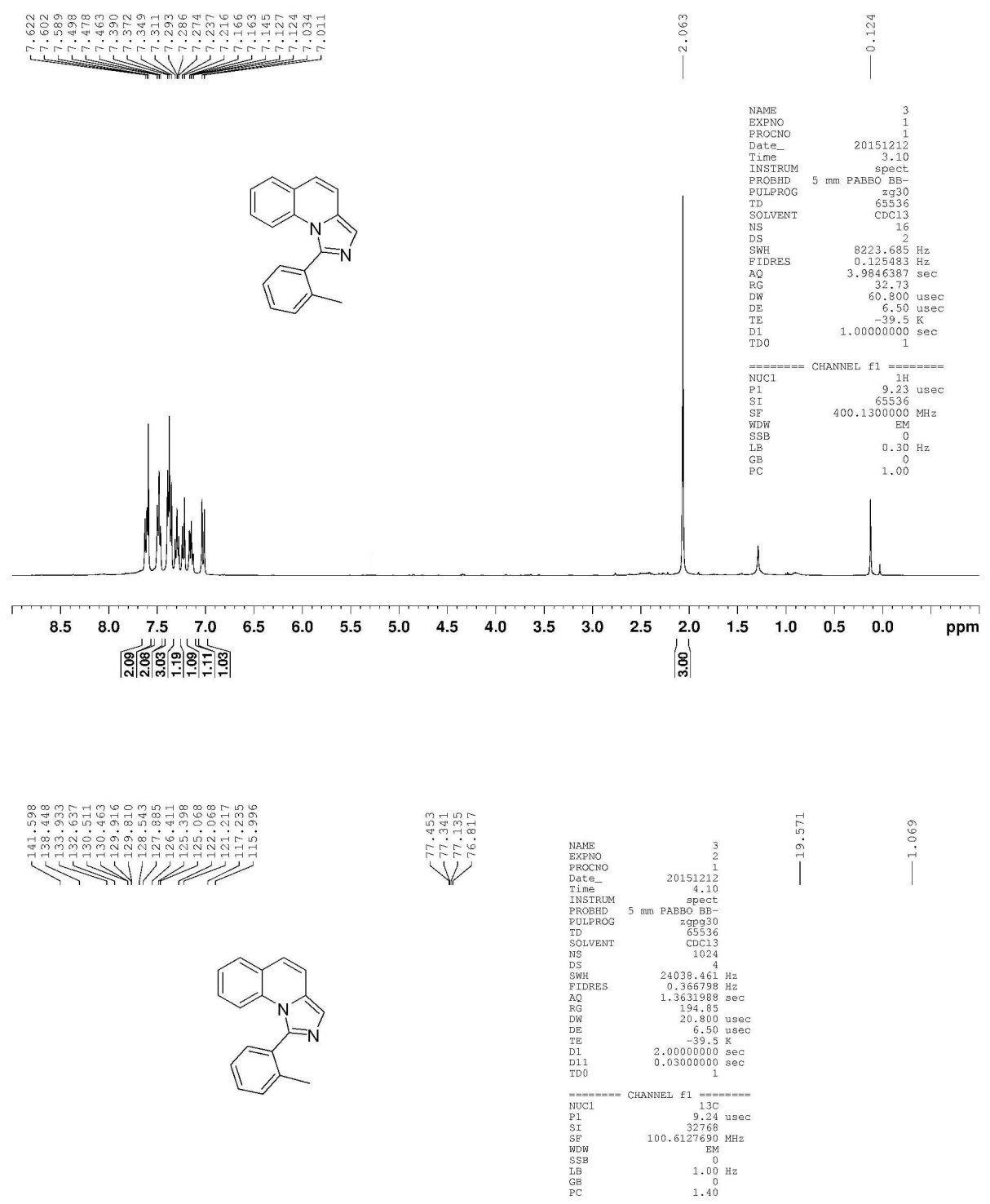
숭ํำ:
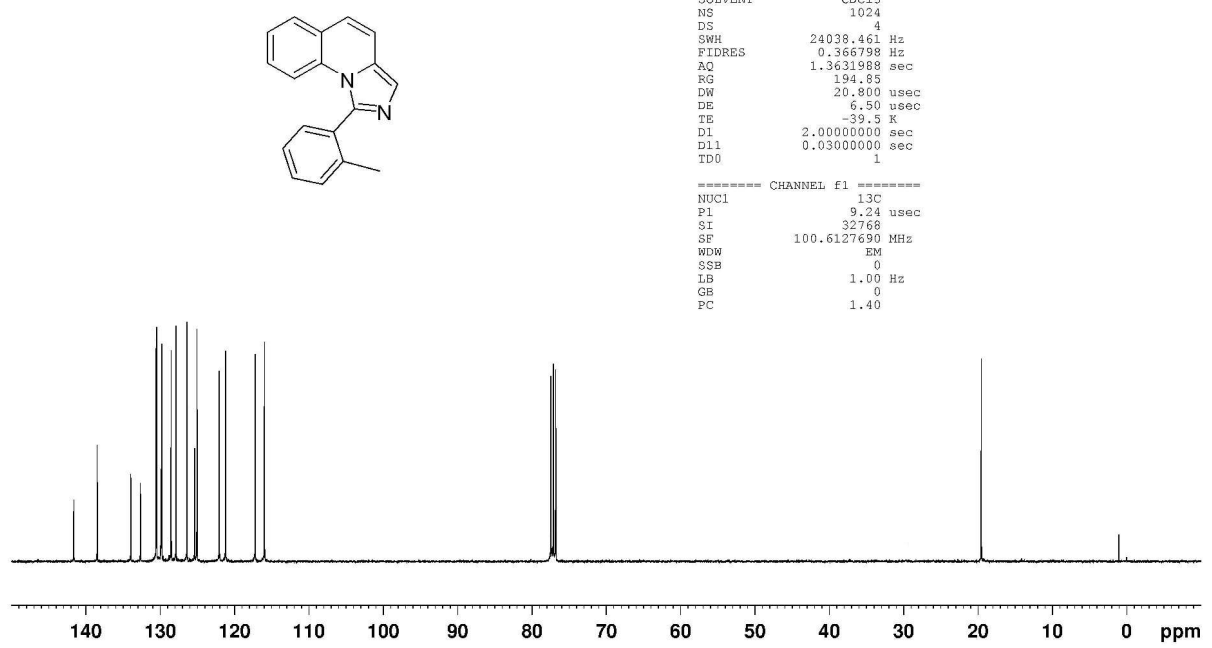
1-(4-methoxyphenyl)imidazo[1,5-a]quinoline (3ae).
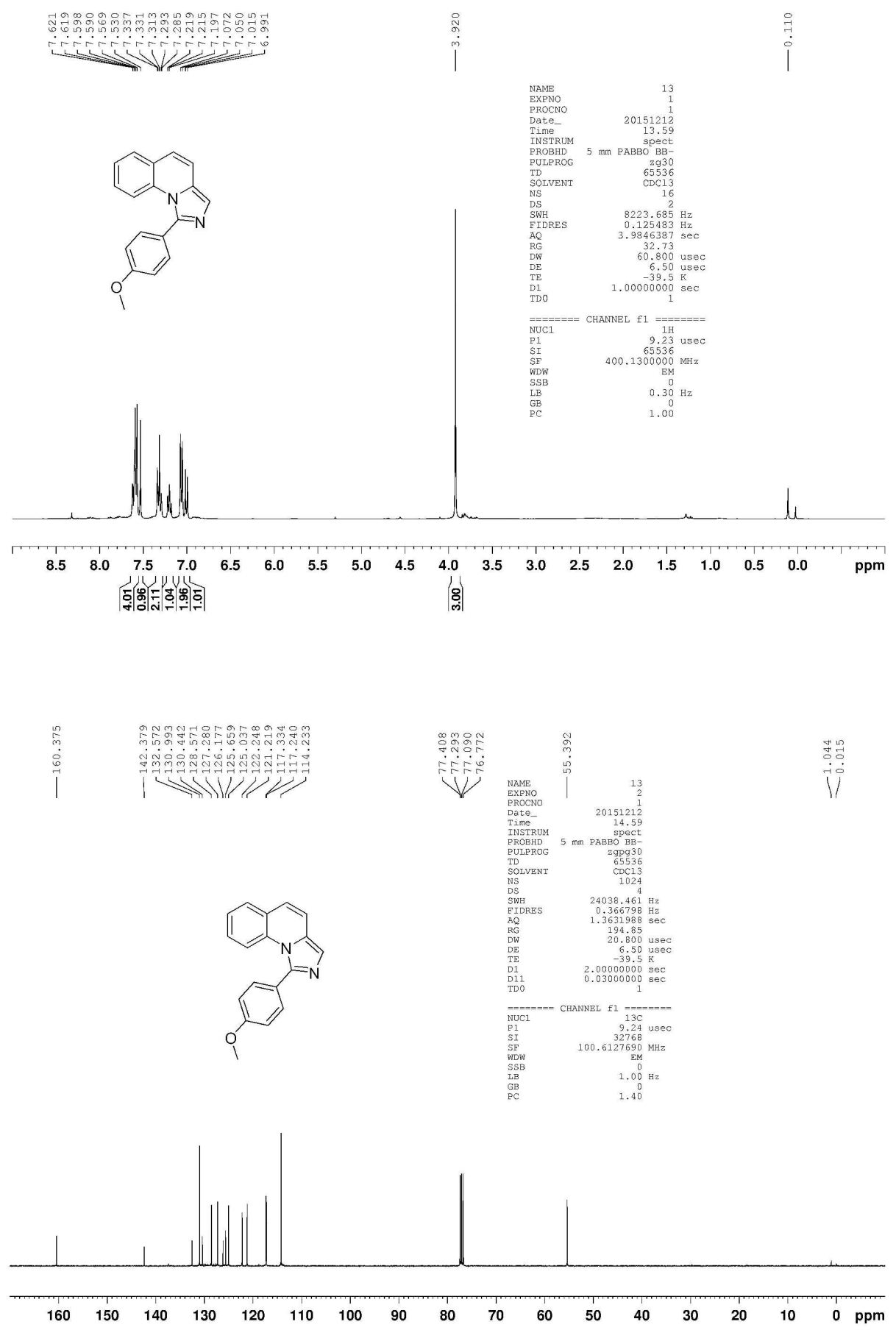
1-(2-methoxyphenyl)imidazo[1,5-a]quinoline (3af).
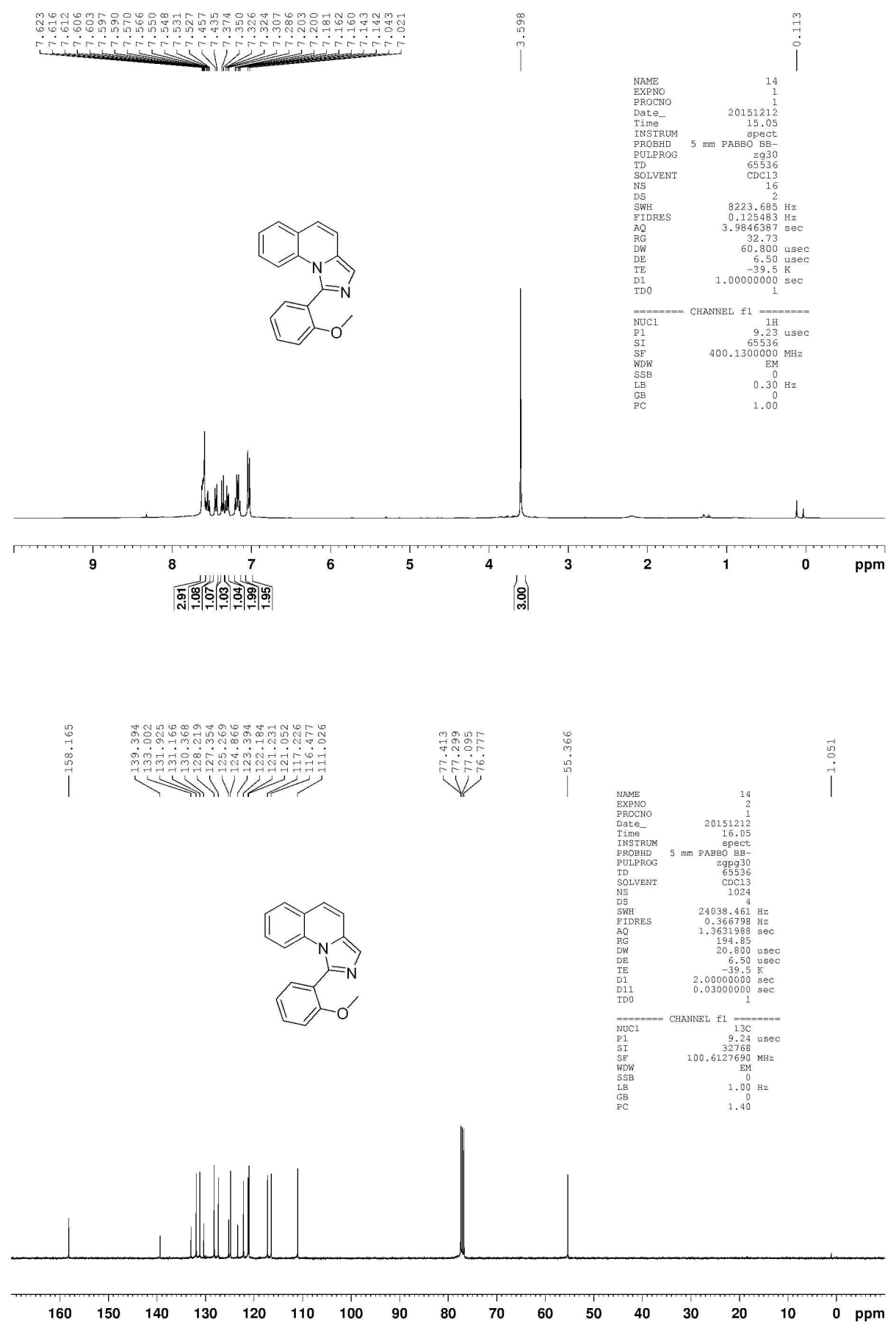
1-(4-(trifluoromethyl)phenyl)imidazo[1,5-a]quinoline (3ag).
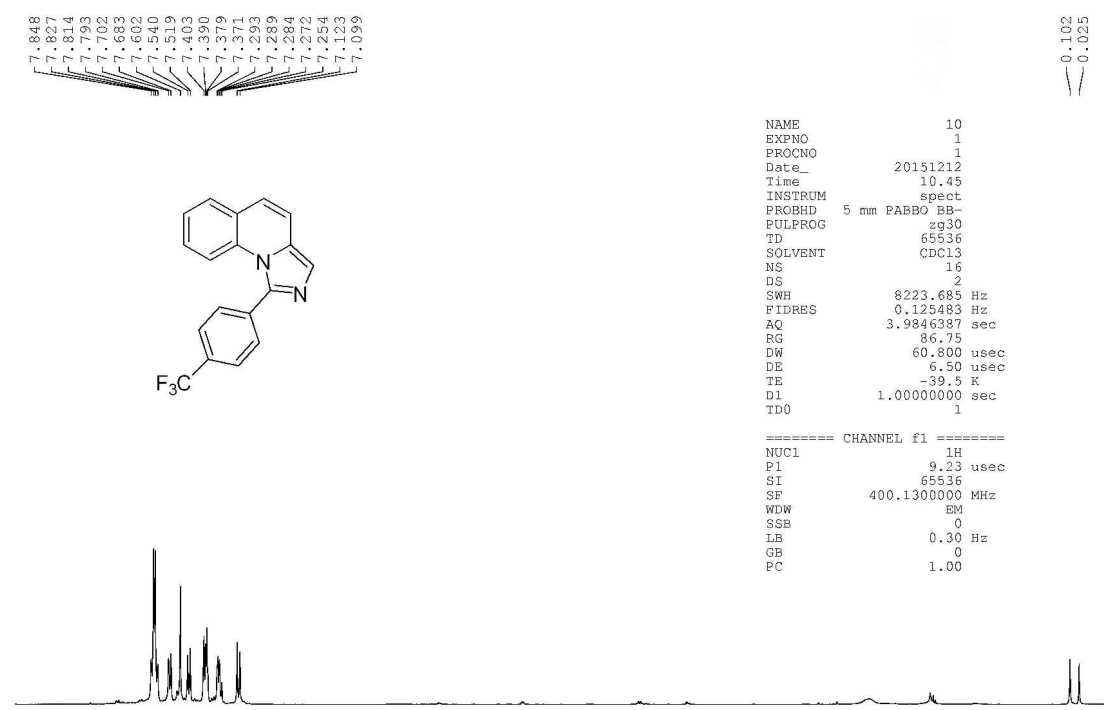

$\begin{array}{lllllllllllllllllllll}8.5 & 8.0 & 7.5 & 7.0 & 6.5 & 6.0 & 5.5 & 5.0 & 4.5 & 4.0 & 3.5 & 3.0 & 2.5 & 2.0 & 1.5 & 1.0 & 0.5 & 0.0 & \mathrm{ppm}\end{array}$

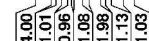

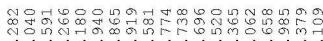
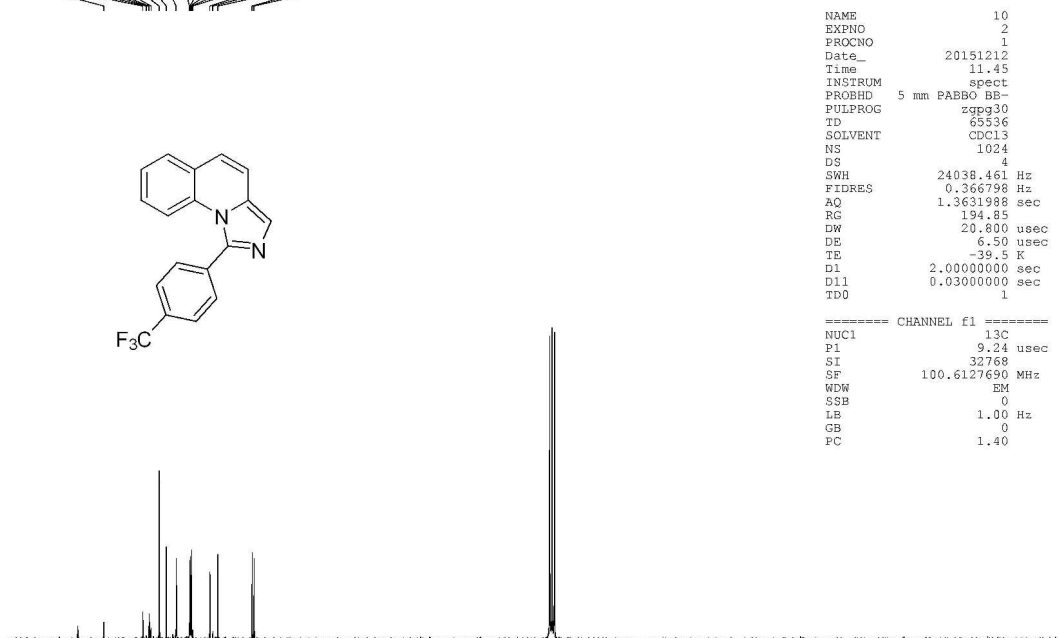

140

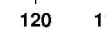

100

90

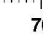

60

$50 \quad 40$

20

$10 \quad 0 \mathrm{ppm}$ 
1-(4-chlorophenyl)imidazo[1,5-a]quinoline (3ah).
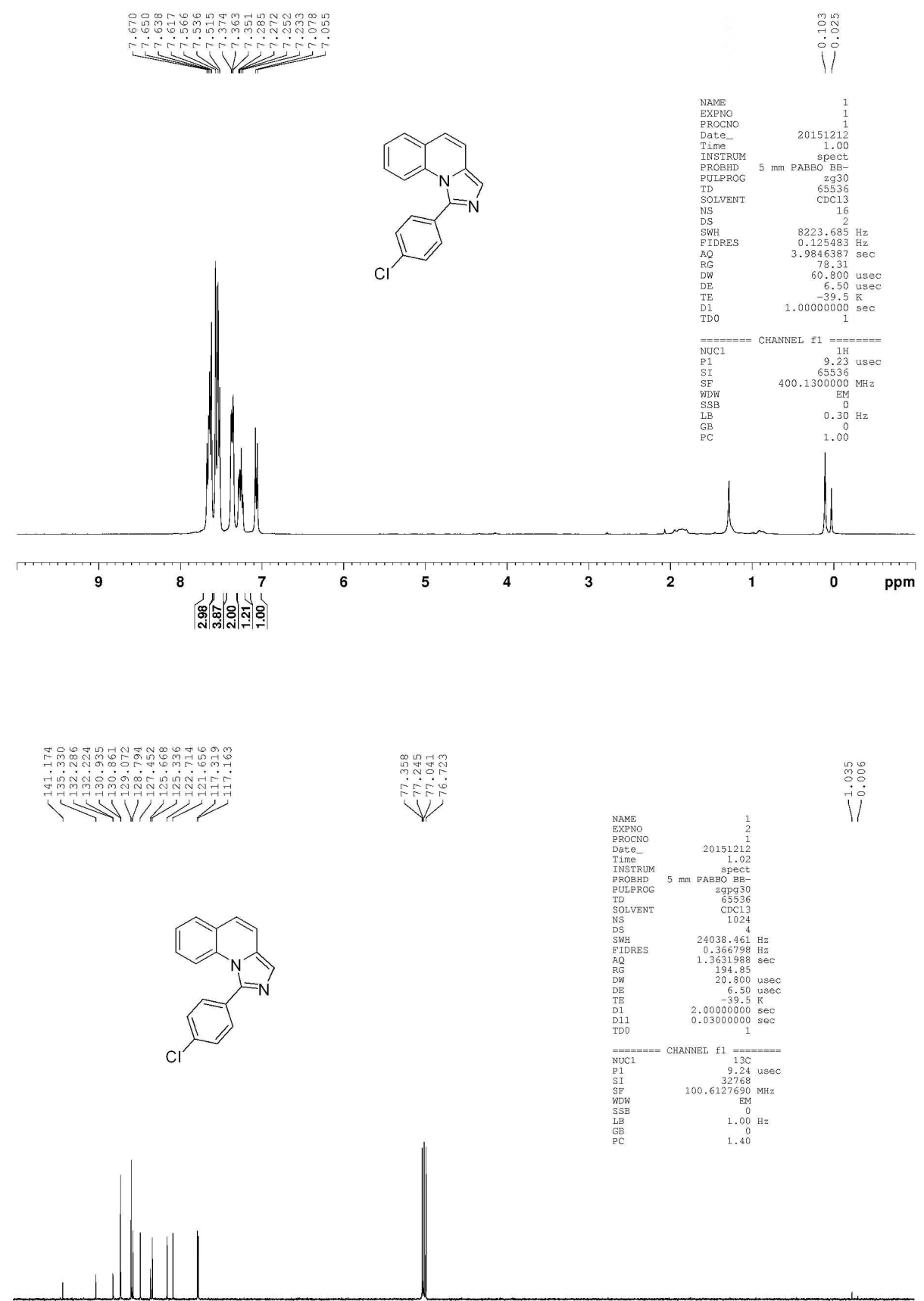

ii

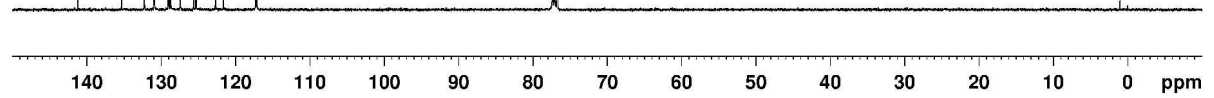


1-(3-chlorophenyl)imidazo[1,5-a]quinoline (3ai).
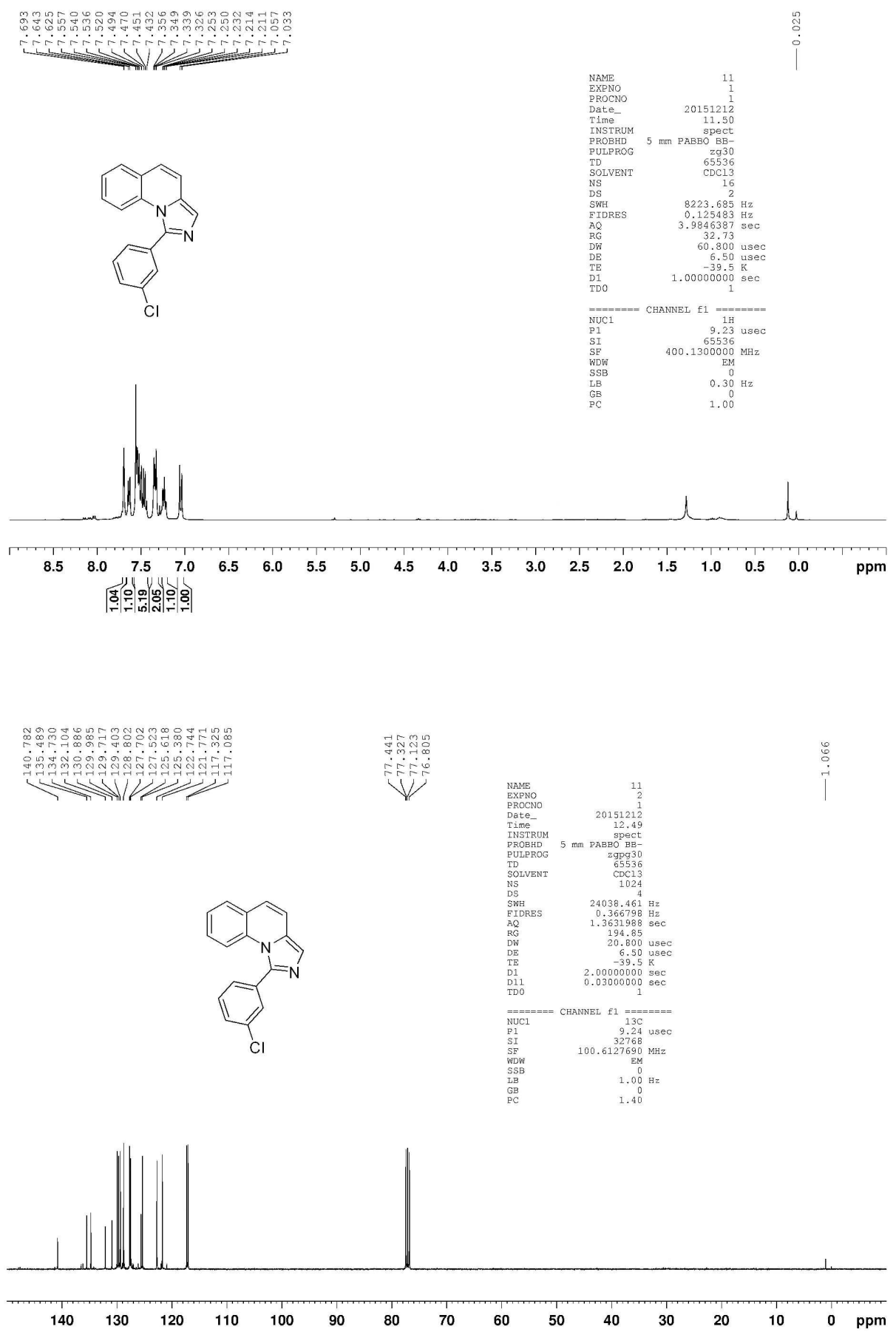
1-(2-chlorophenyl)imidazo[1,5-a]quinoline (3aj).
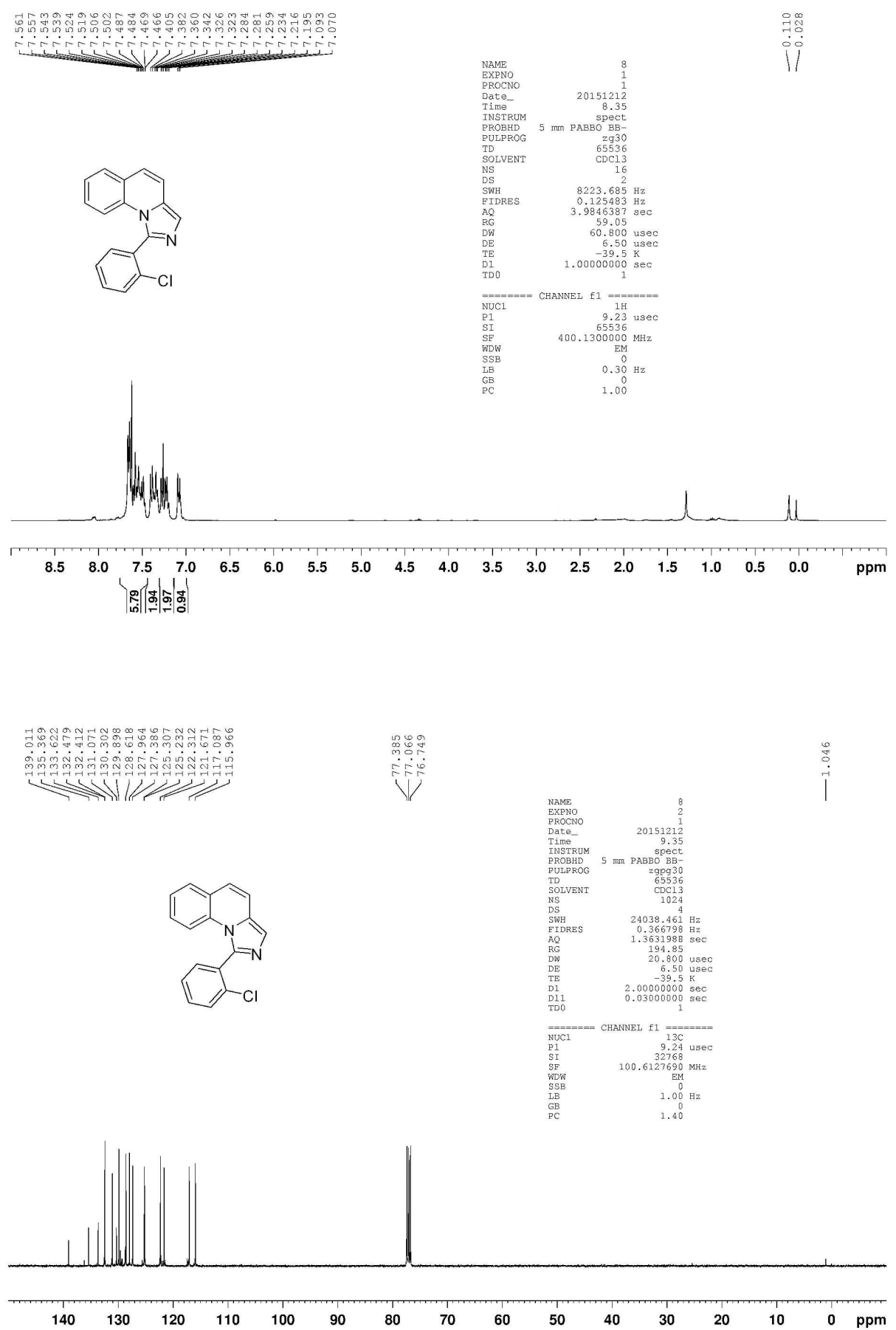
1-(3-fluorophenyl)imidazo[1,5-a]quinoline (3ak).
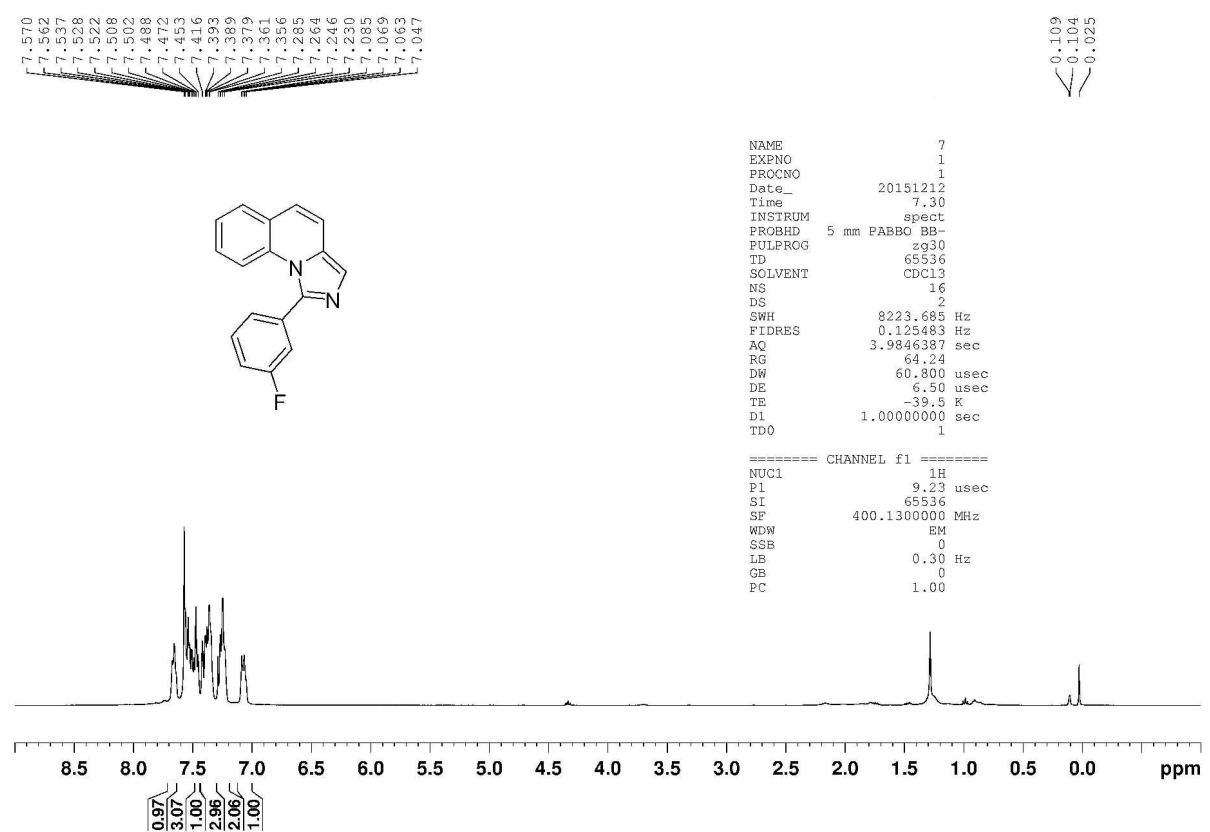

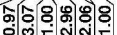
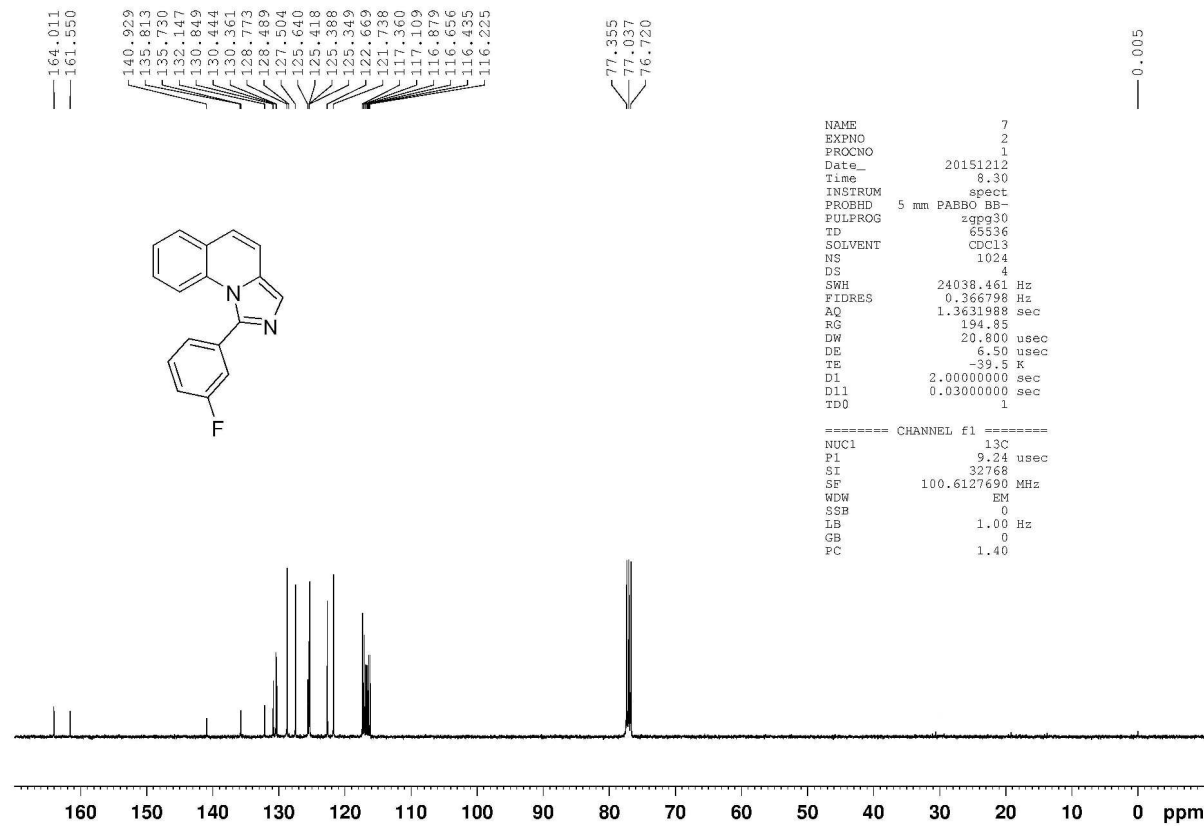
1-(2-fluorophenyl)imidazo[1,5-a]quinoline (3al).
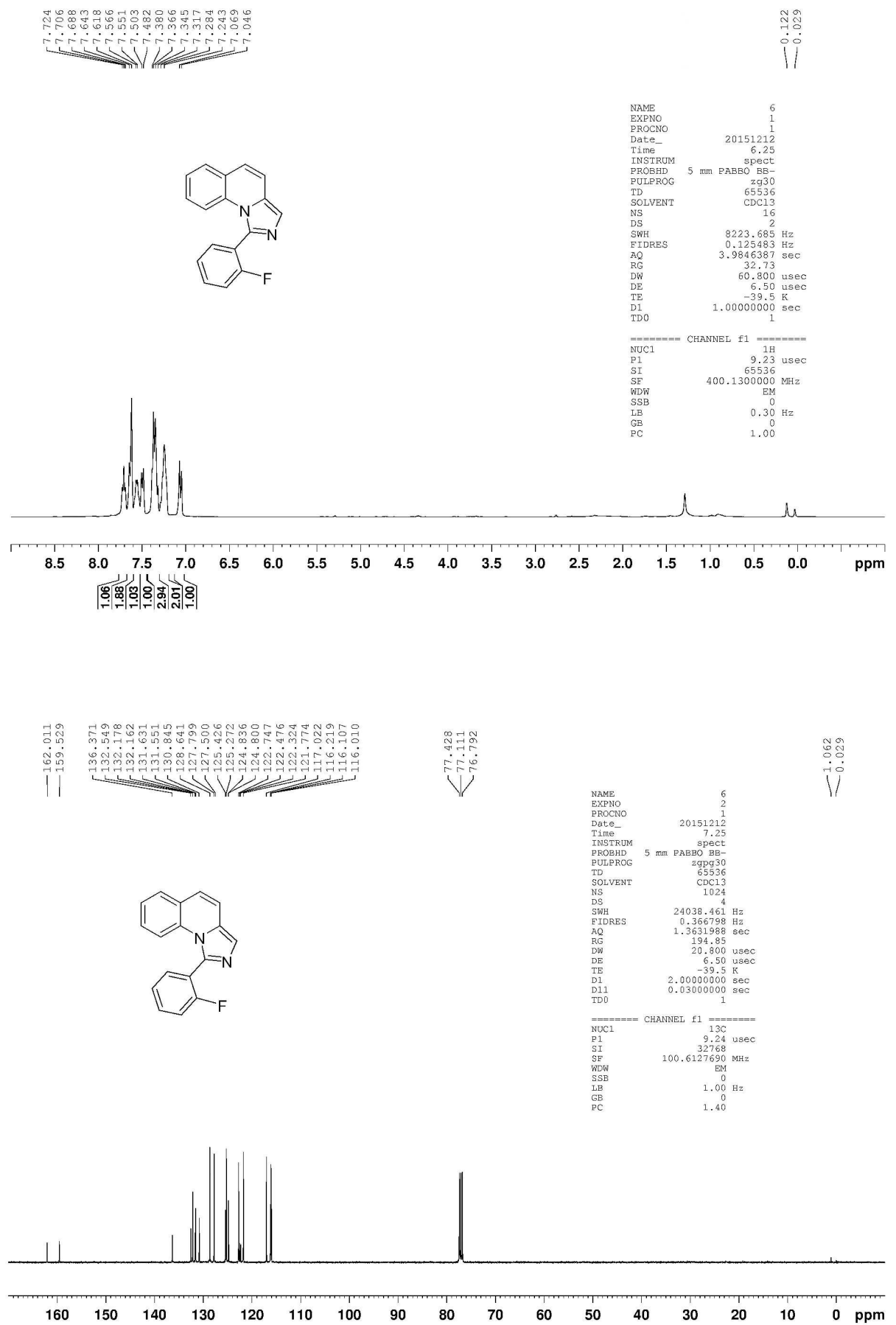
1-(4-bromophenyl)imidazo[1,5-a]quinoline (3am).
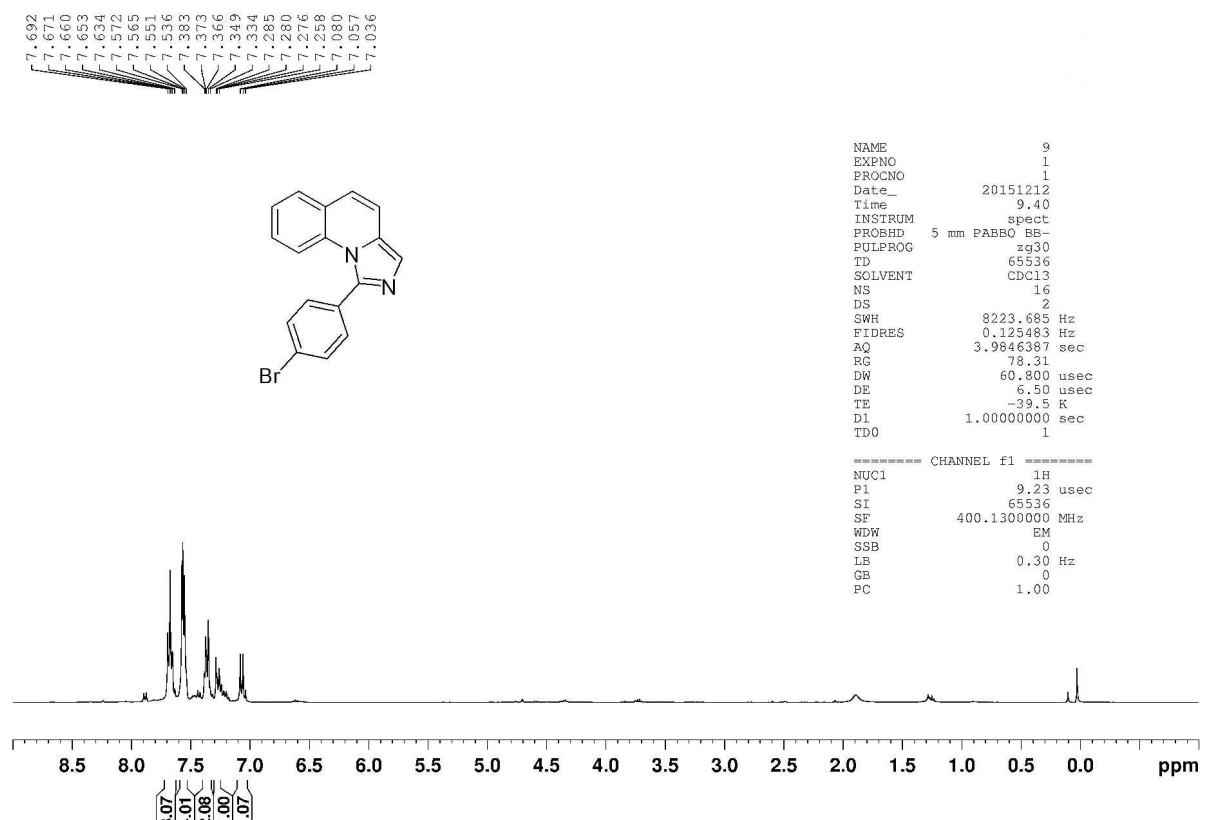

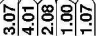

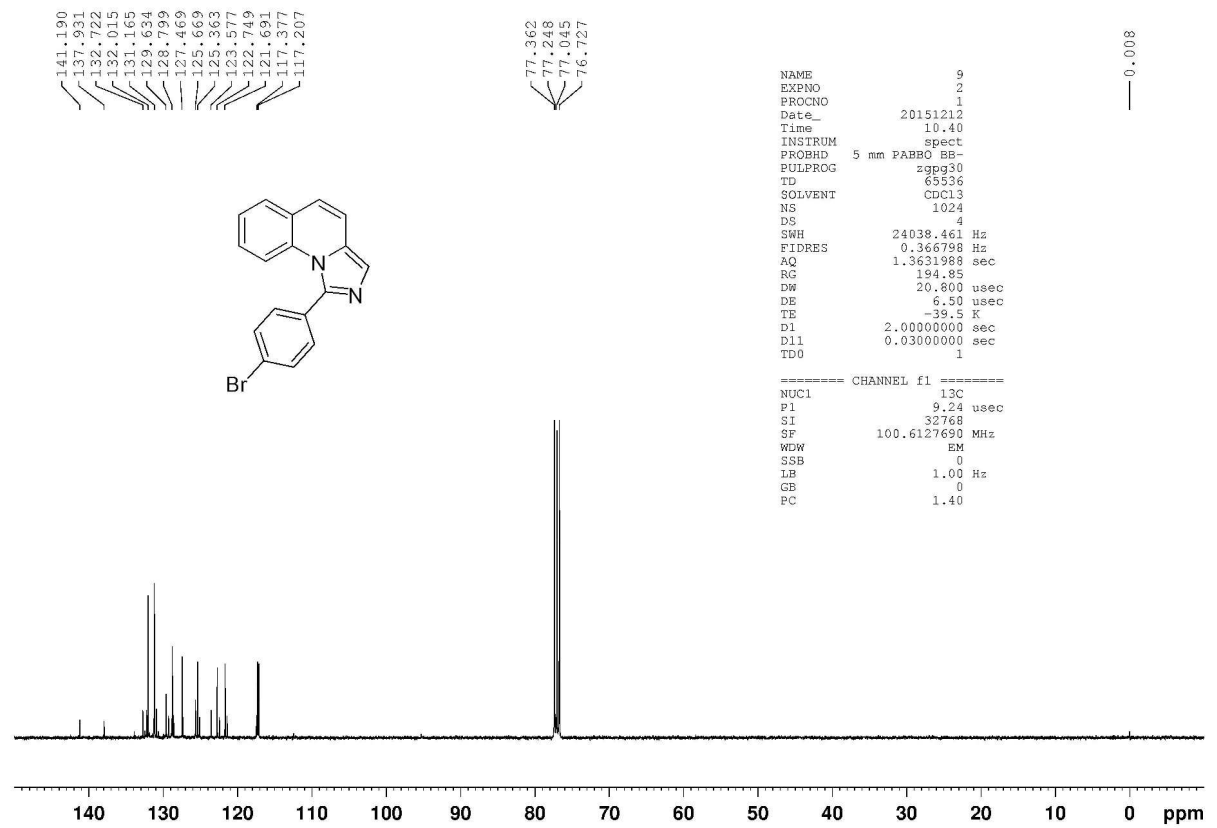


1-(benzo[d][1,3]dioxol-5-yl)imidazo[1,5- $a]$ quinoline (3an).
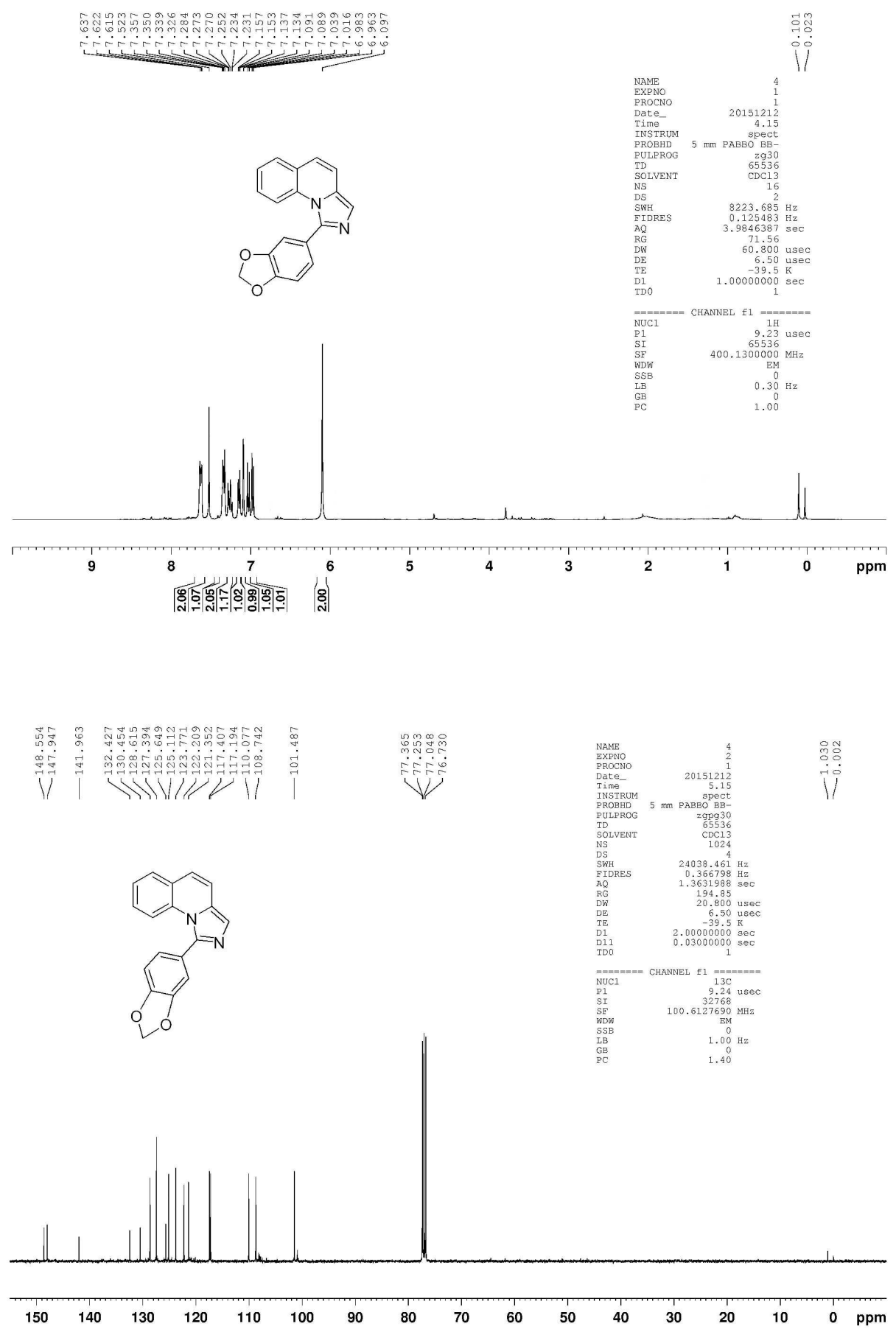
1-(naphthalen-1-yl)imidazo[1,5-a]quinoline (3ao).

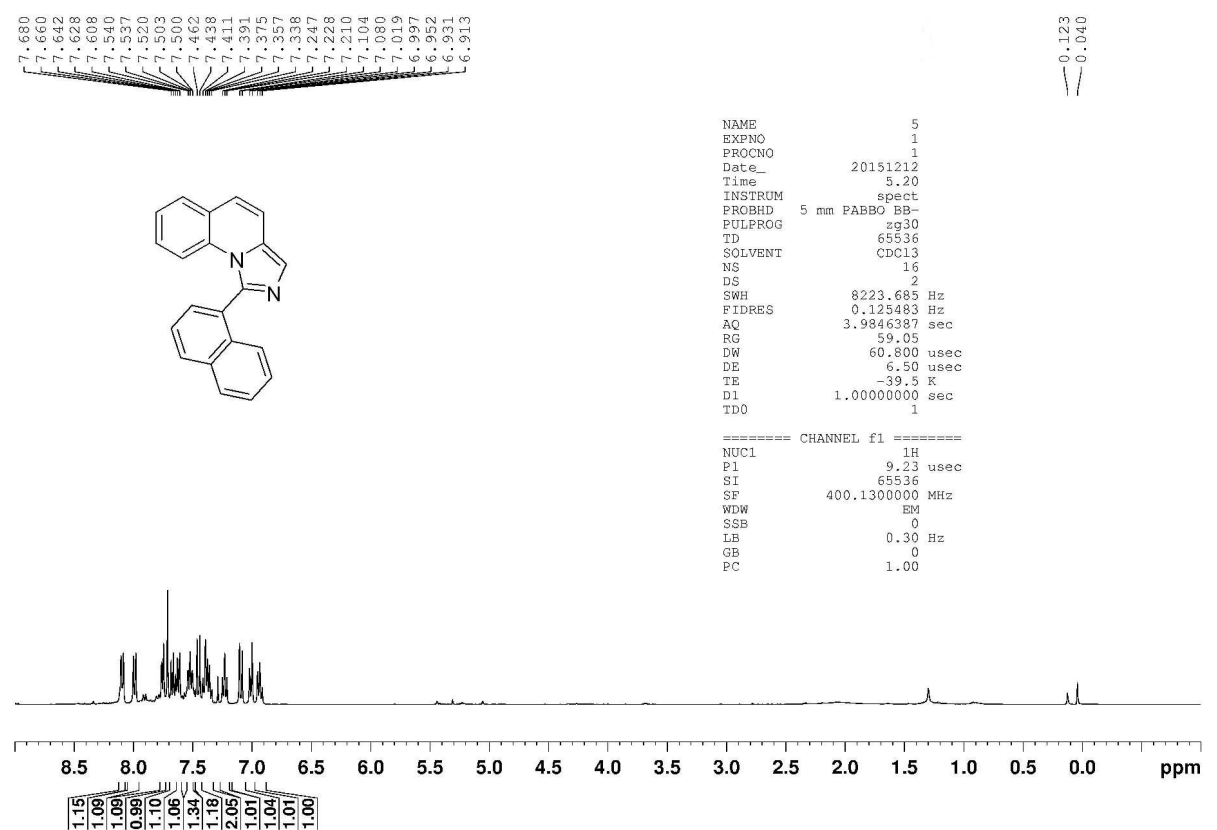

To:
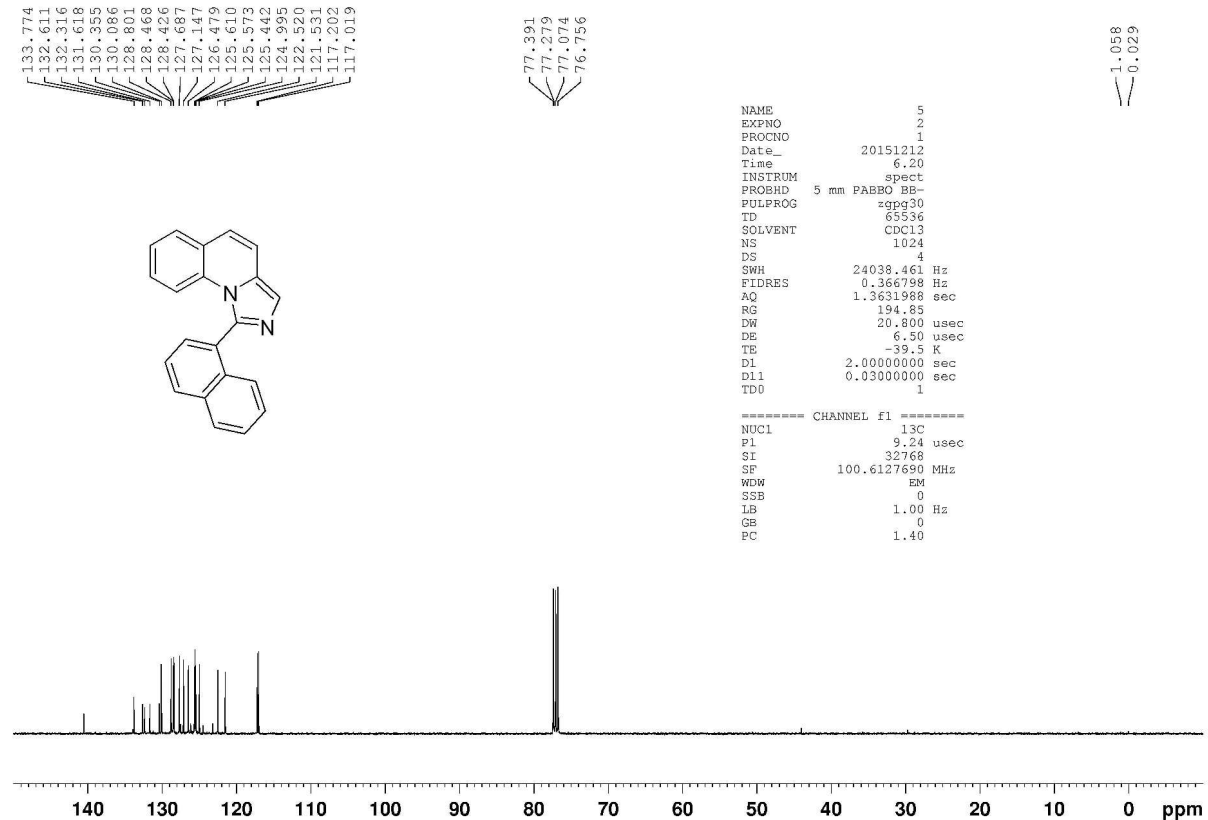
1-(furan-2-yl)imidazo[1,5-a]quinoline (3ap).

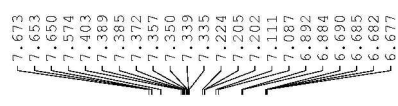

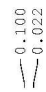
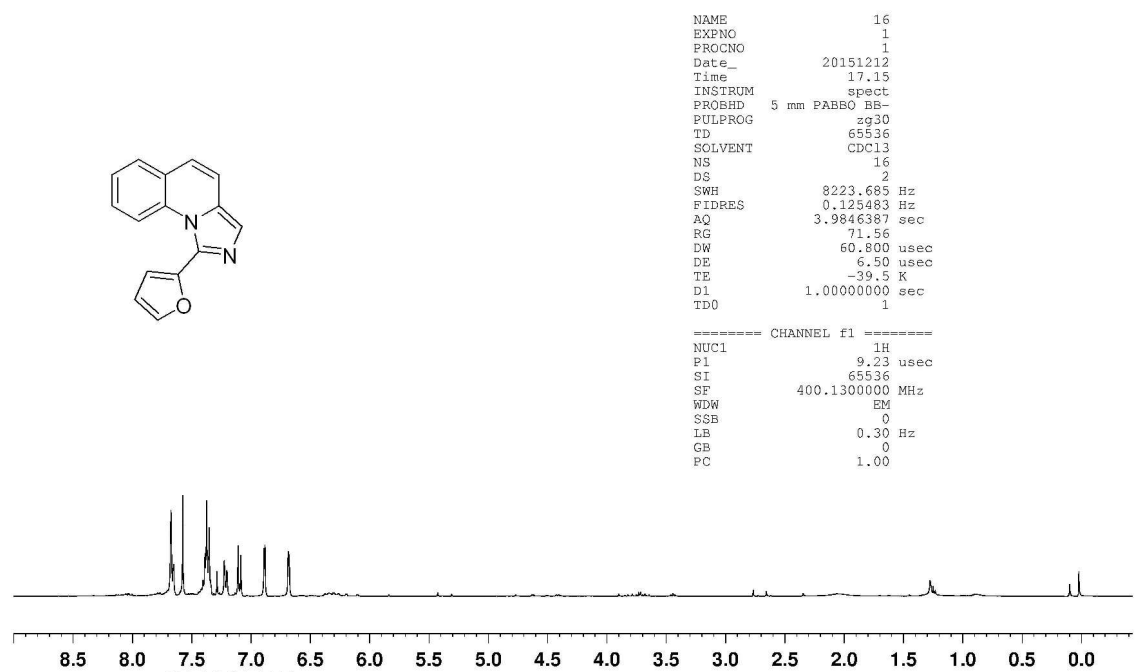
두으.
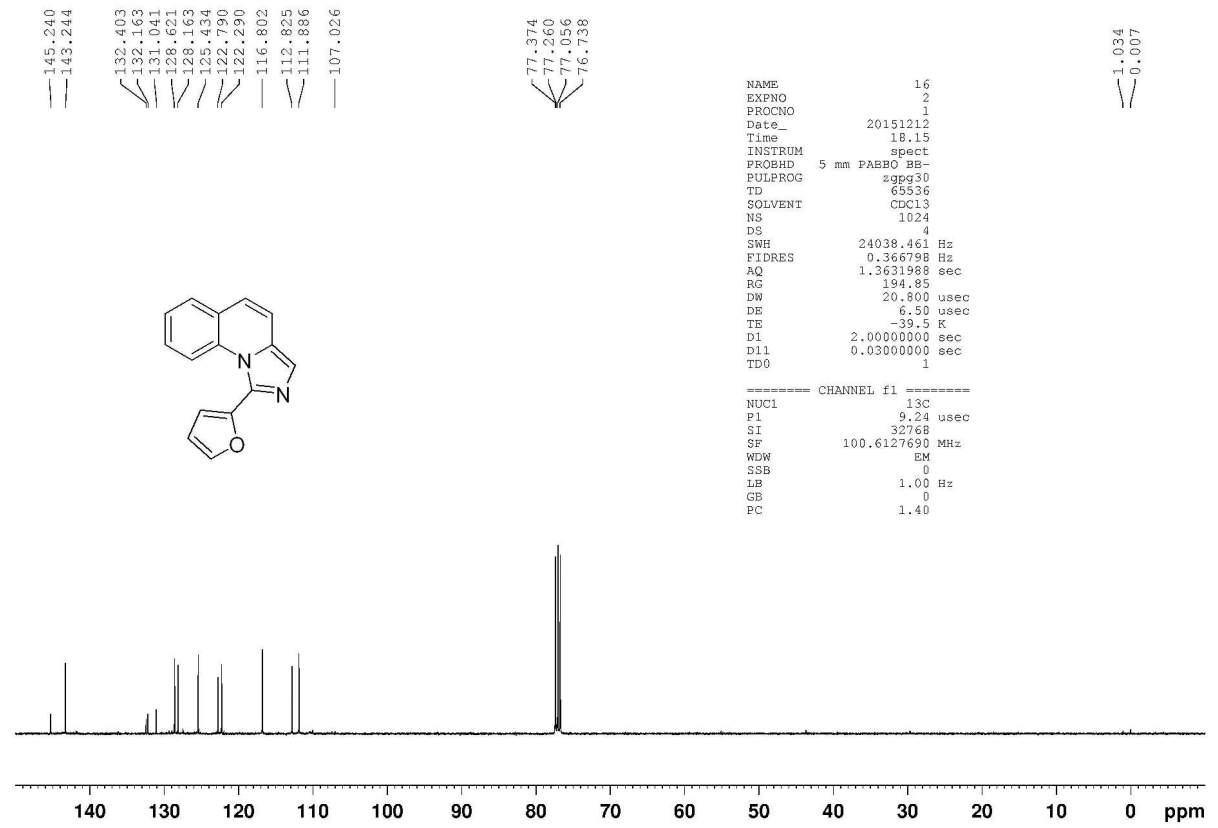
1-ethylimidazo[1,5-a]quinoline (3aq)
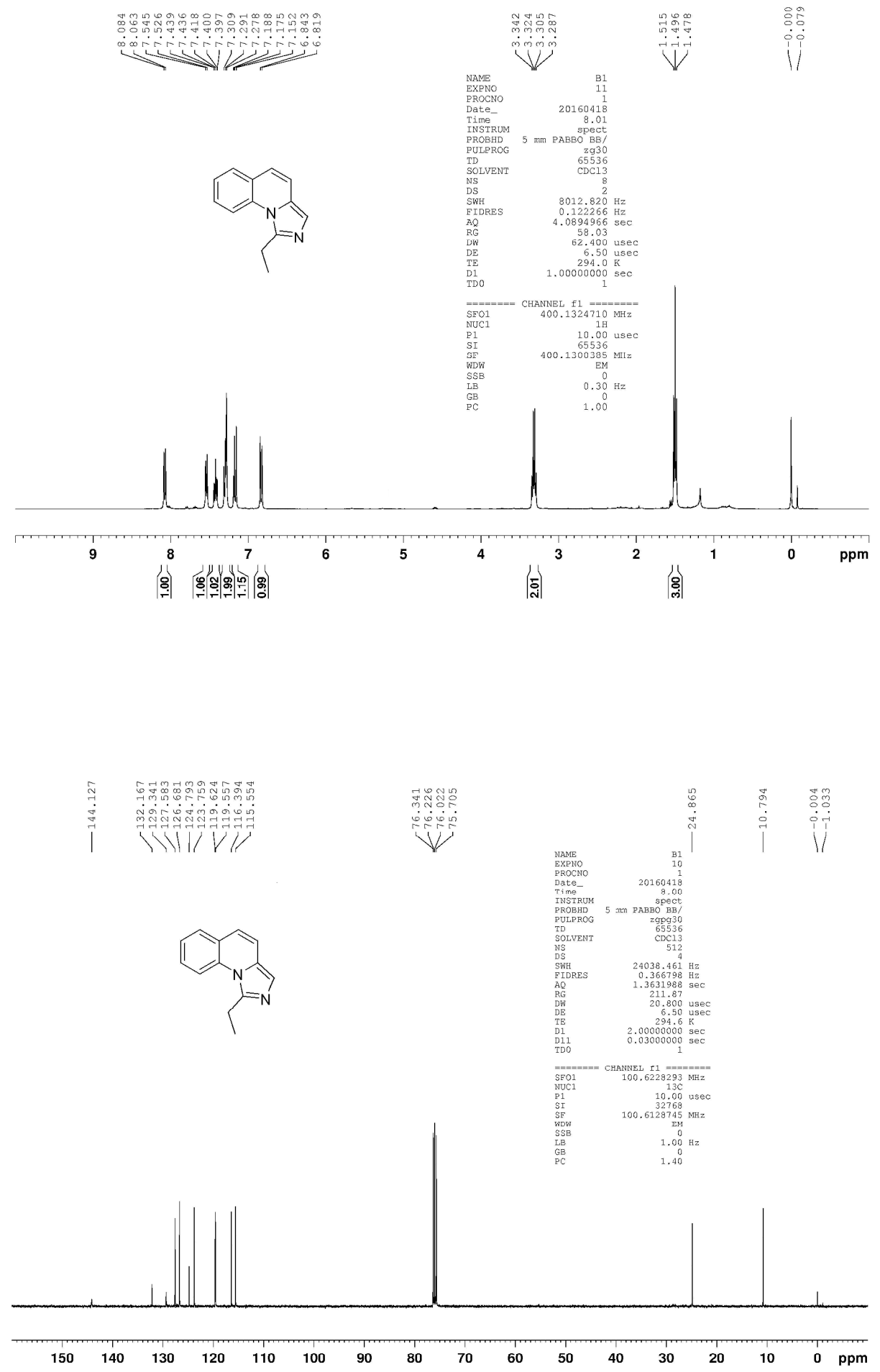
7-methyl-1-phenylimidazo[1,5-a]quinoline (3ba).
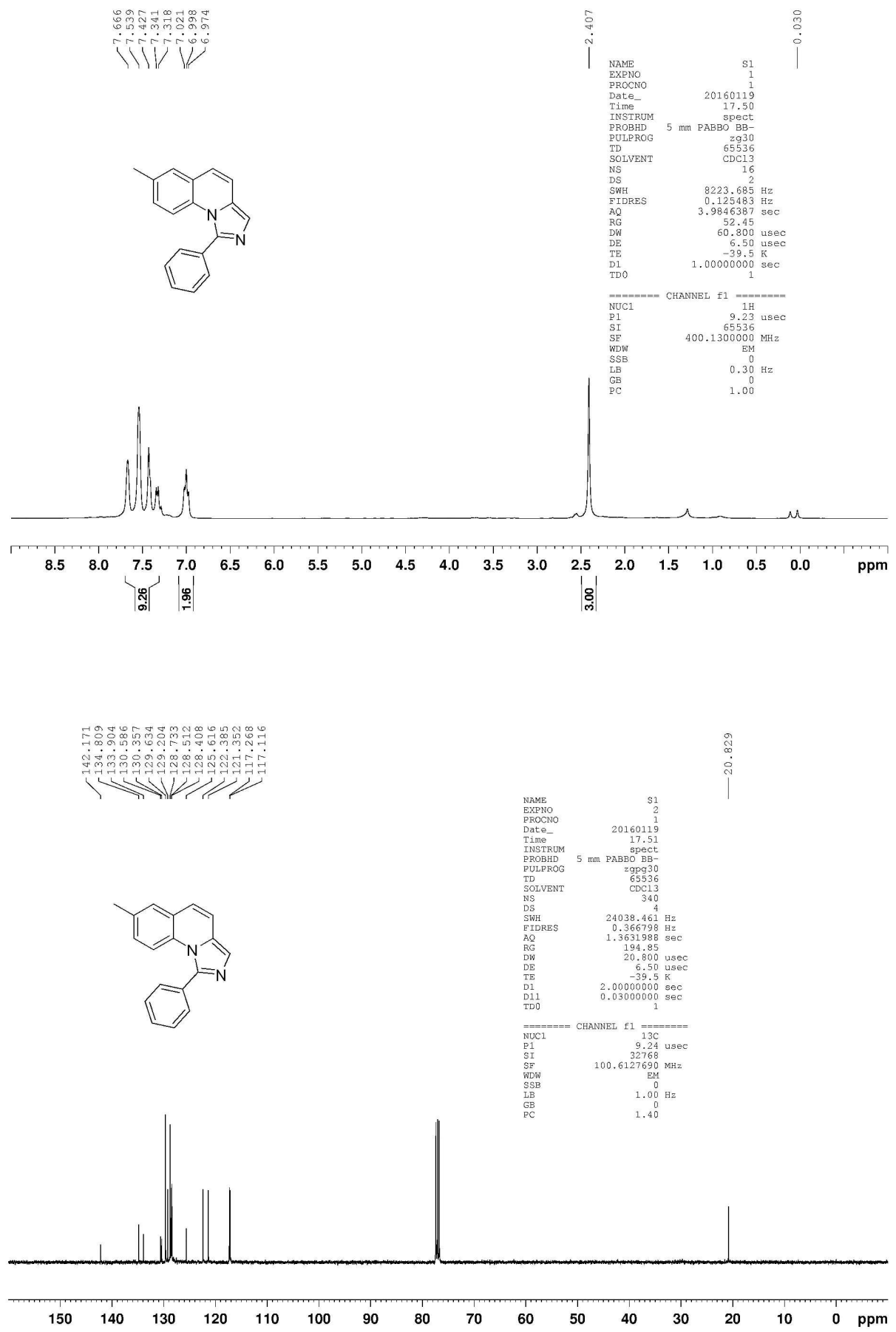
6,8-dimethyl-1-phenylimidazo[1,5-a]quinoline (3ca).
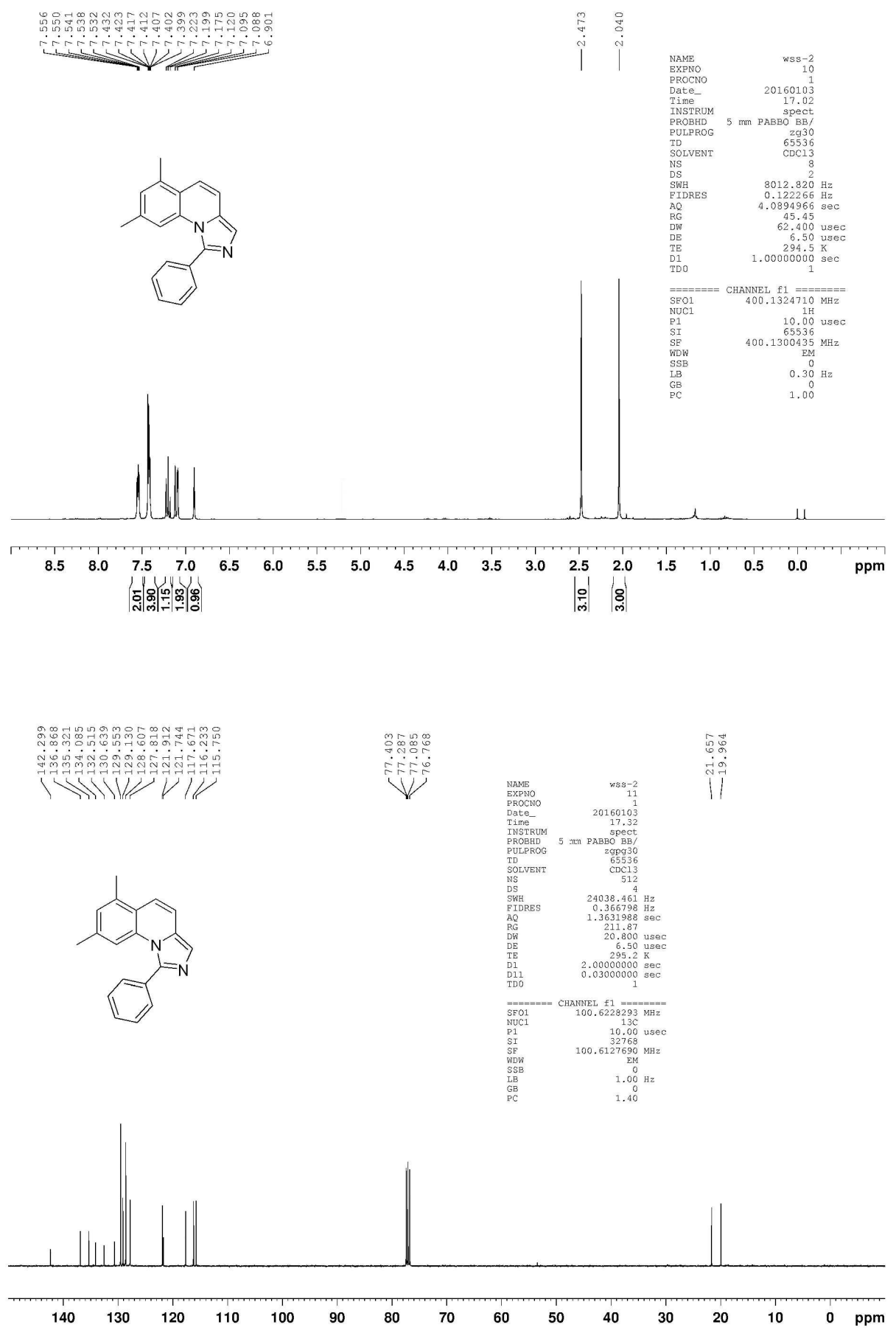
7-methoxy-1-phenylimidazo[1,5- $a$ ]quinoline (3da).

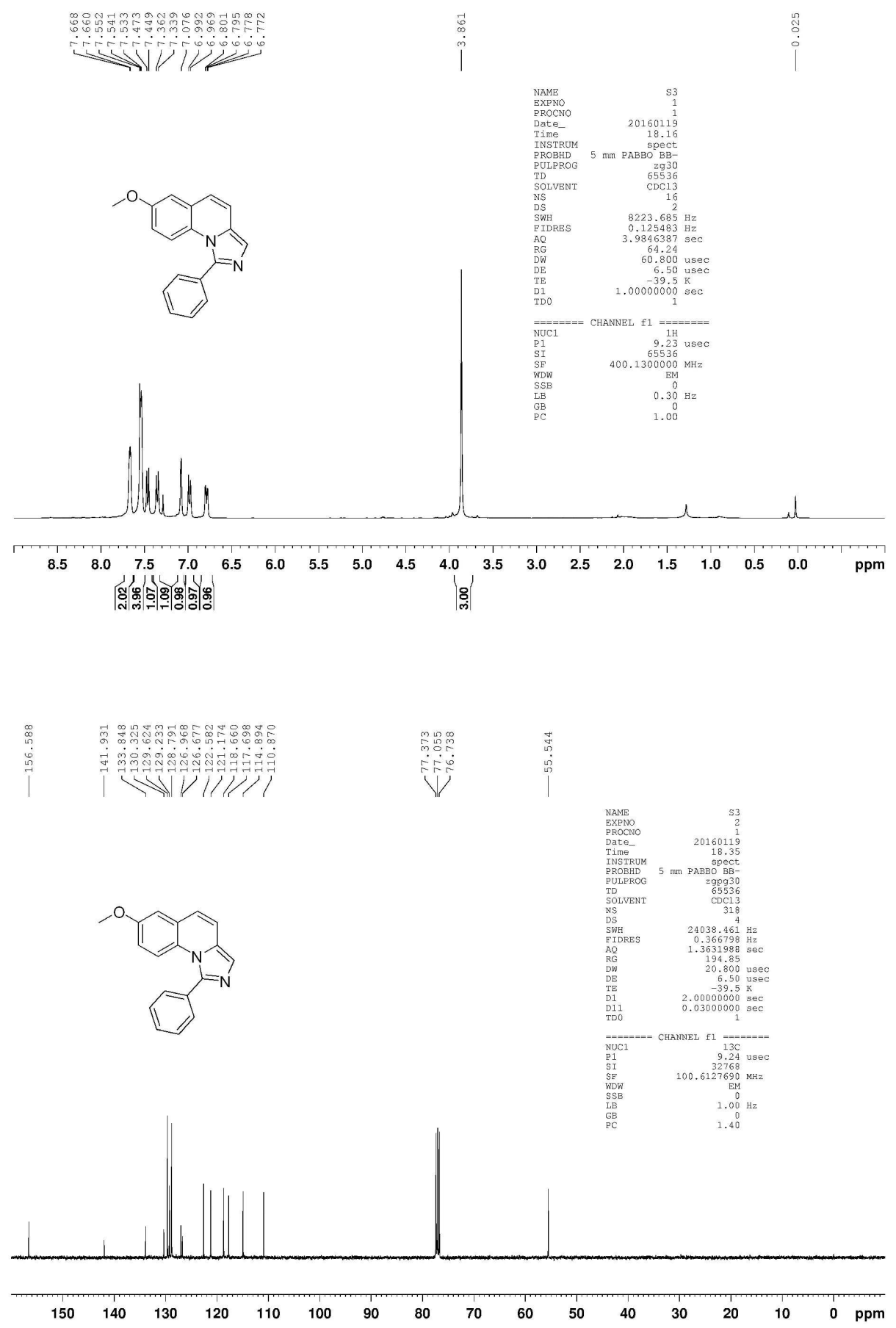


7-ethoxy-1-phenylimidazo[1,5-a]quinoline (3ea).
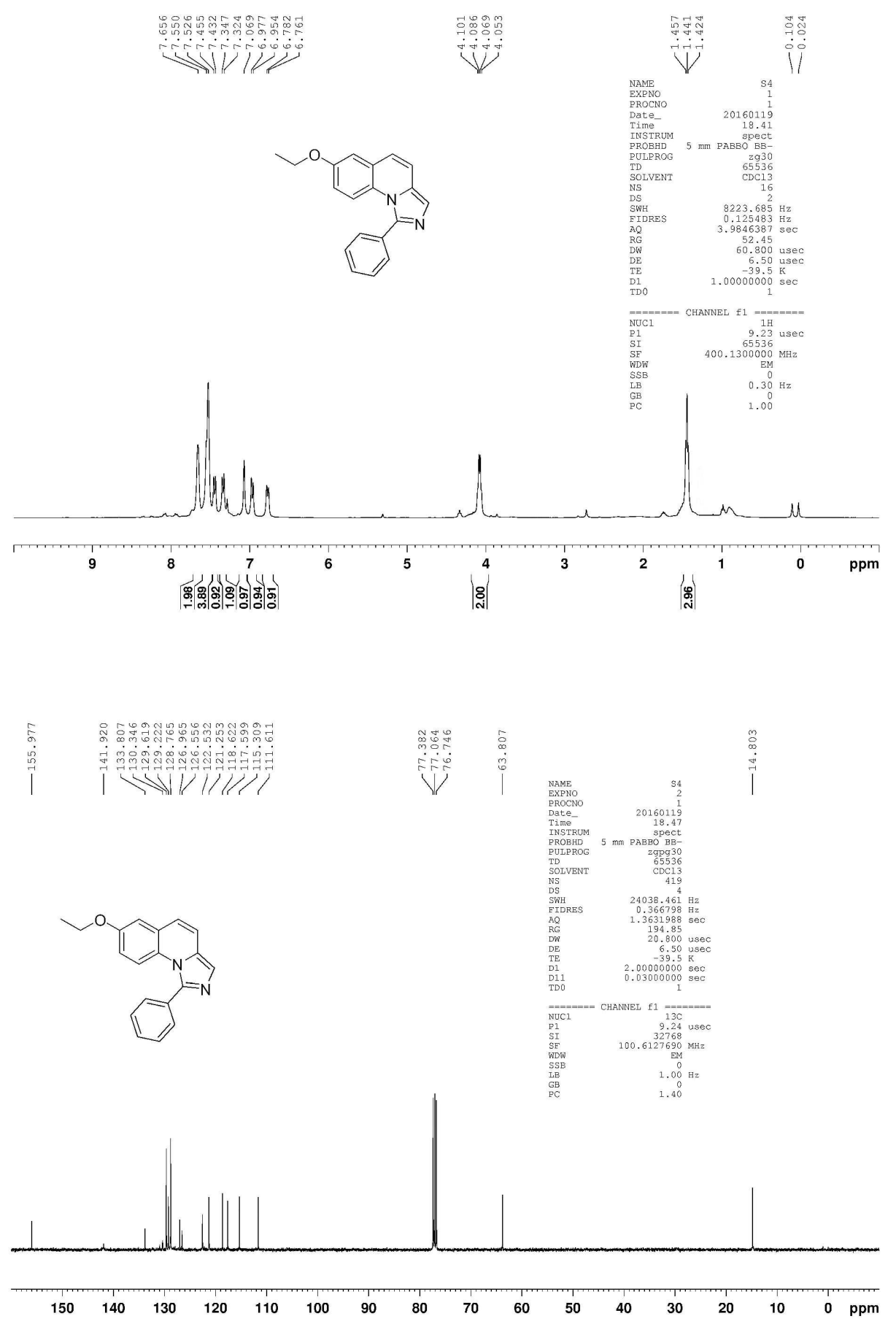
9-methoxy-1-phenylimidazo[1,5-a]quinoline (3fa).
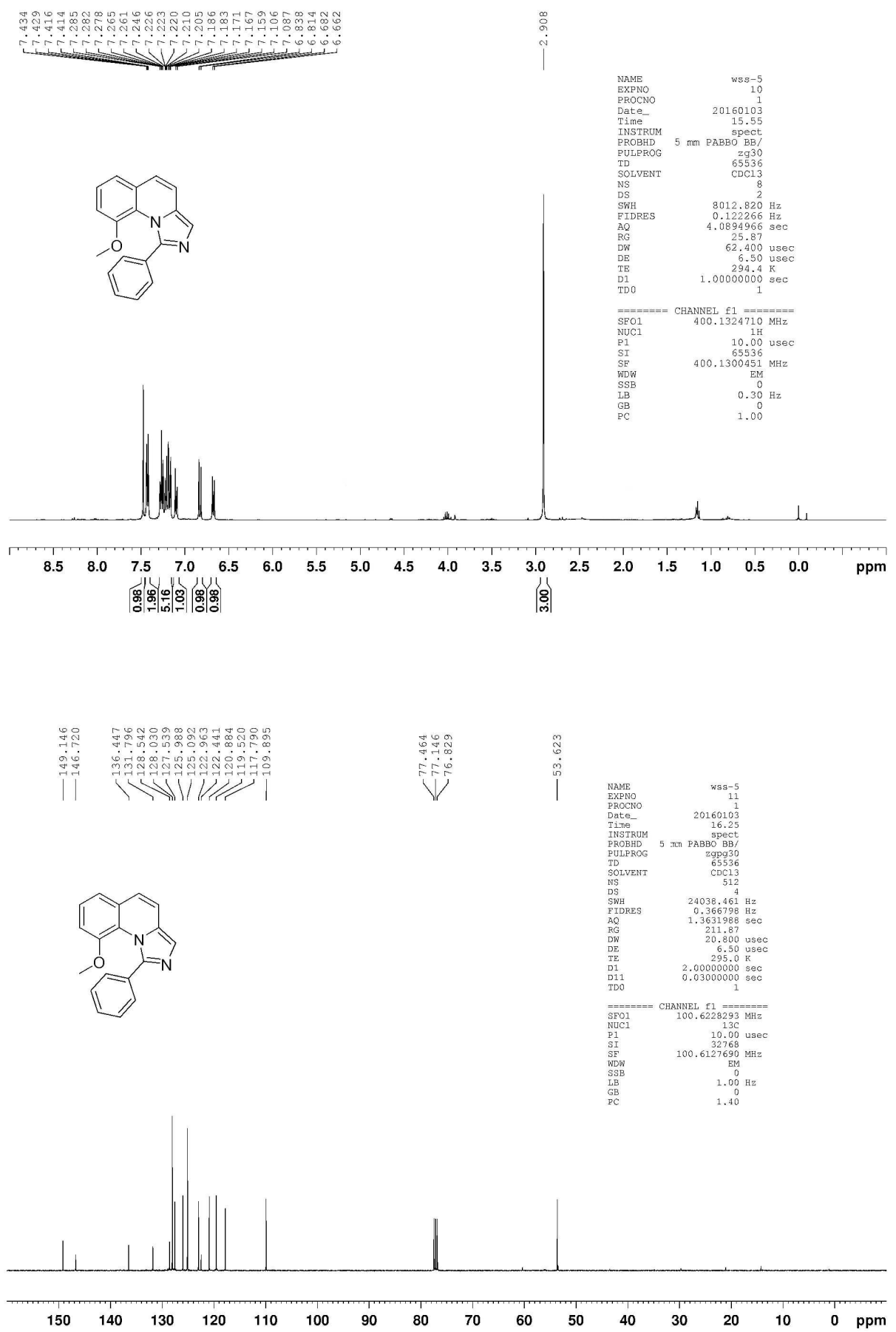
1,5-diphenylimidazo[1,5- $a$ ]quinoline (3ga).
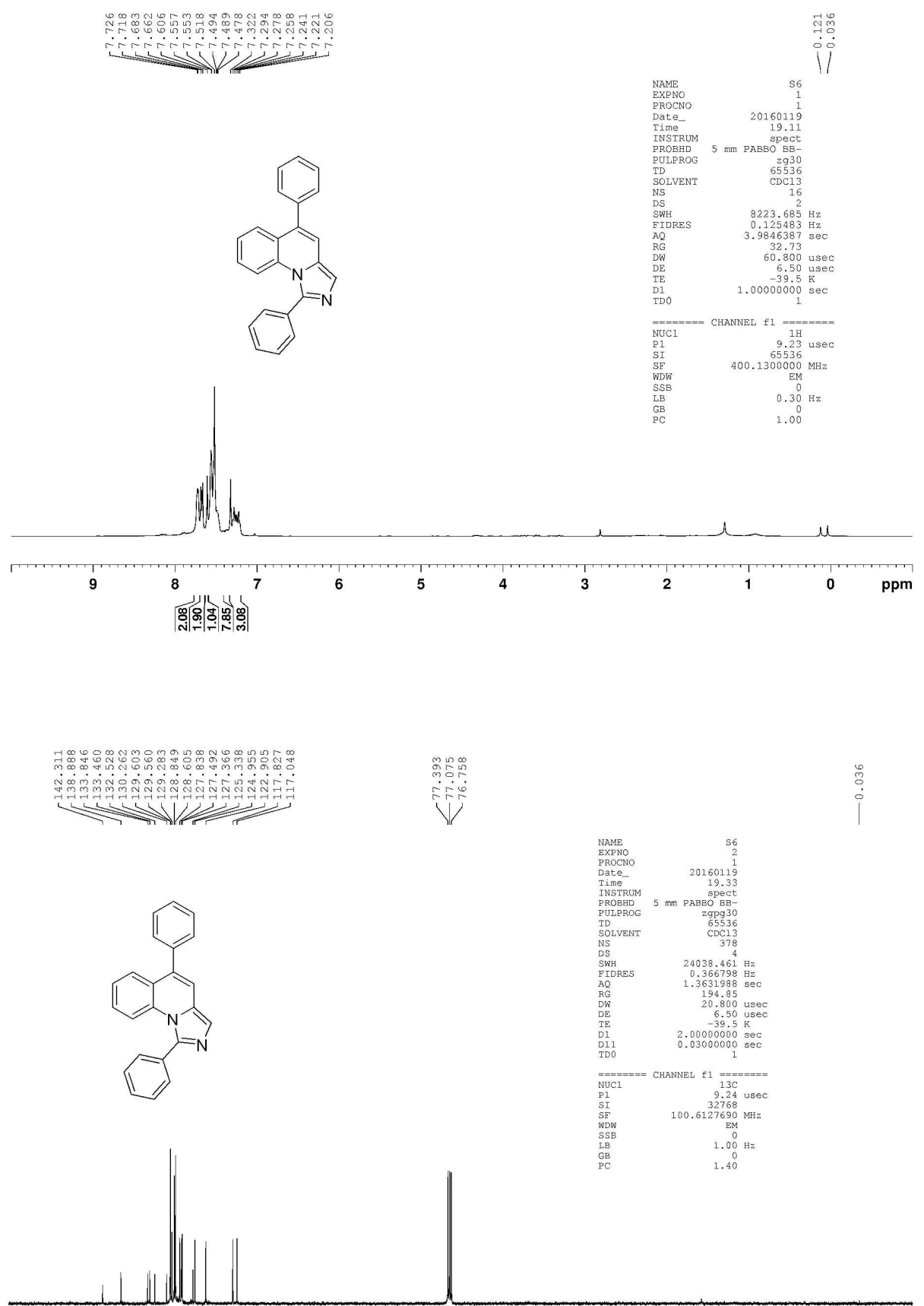
7-chloro-1-phenylimidazo[1,5-a]quinoline (3ha).

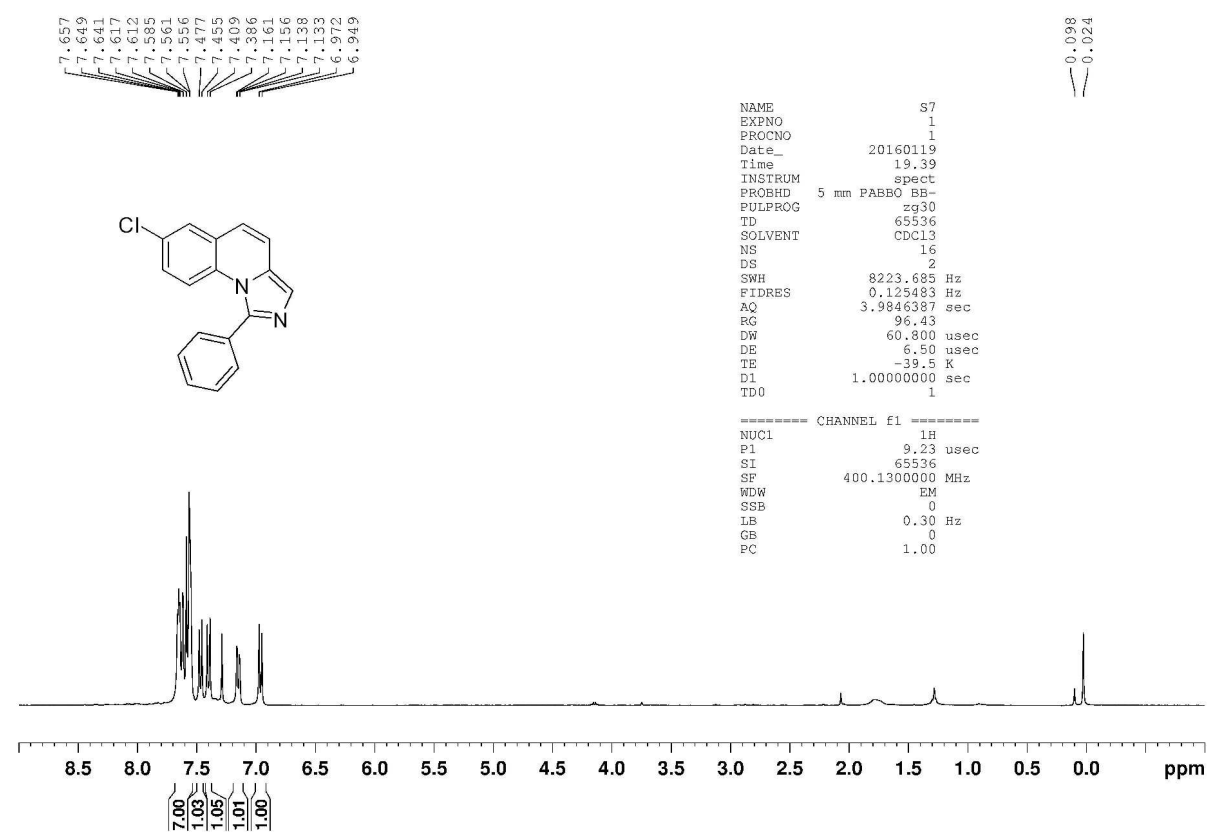

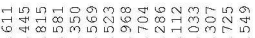

ㅂ.

(lint)

$\sqrt{2}$
$\sqrt{1}$
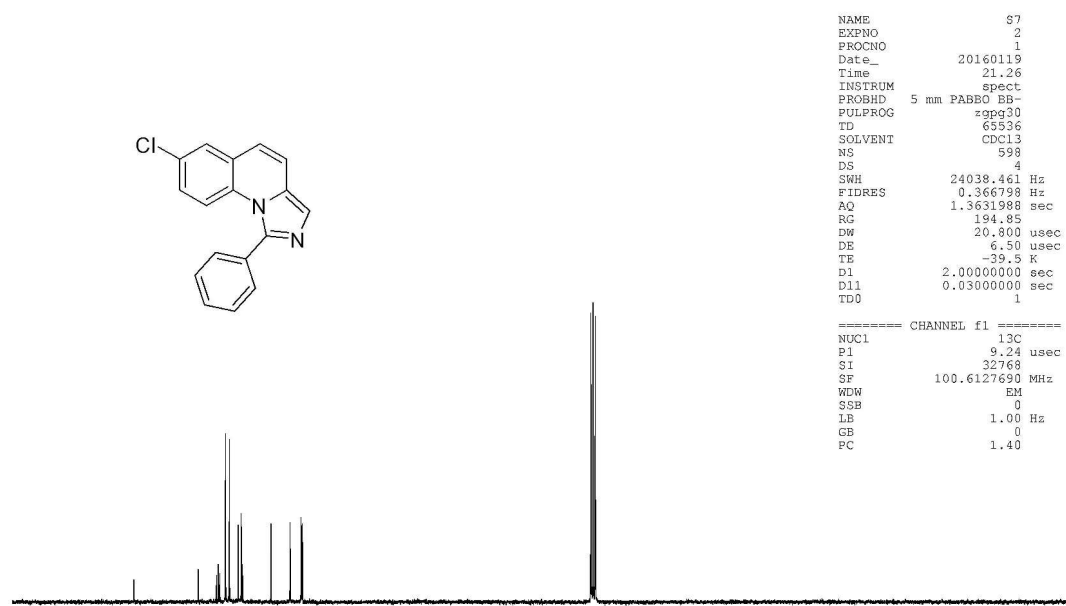

150

$140 \quad 130$

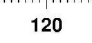

100

80

70

5040

30

$20 \quad 10 \quad 0 \quad \mathrm{ppm}$ 
7-bromo-1-phenylimidazo[1,5-a]quinoline (3ia).
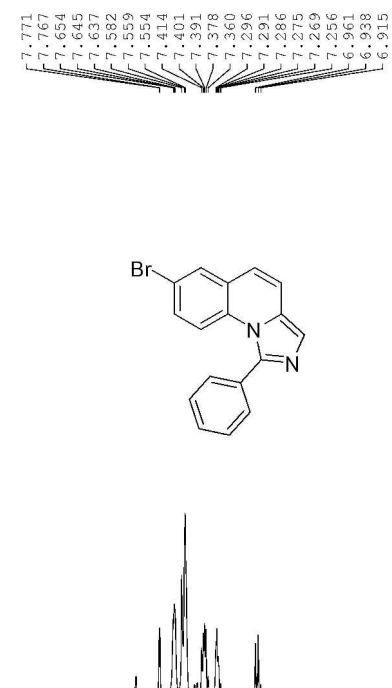
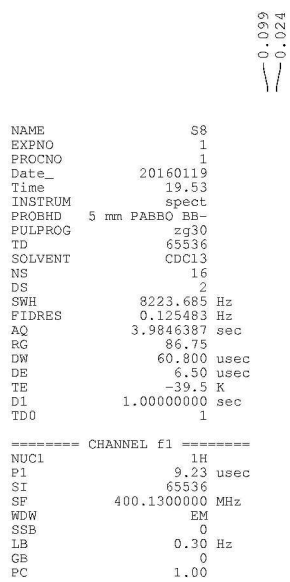

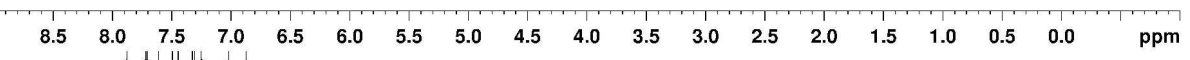
(8)

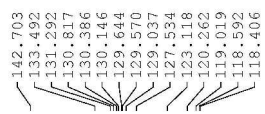

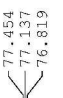<smiles>Brc1ccc2c(ccc3ncc(-c4ccccc4)n32)c1</smiles>
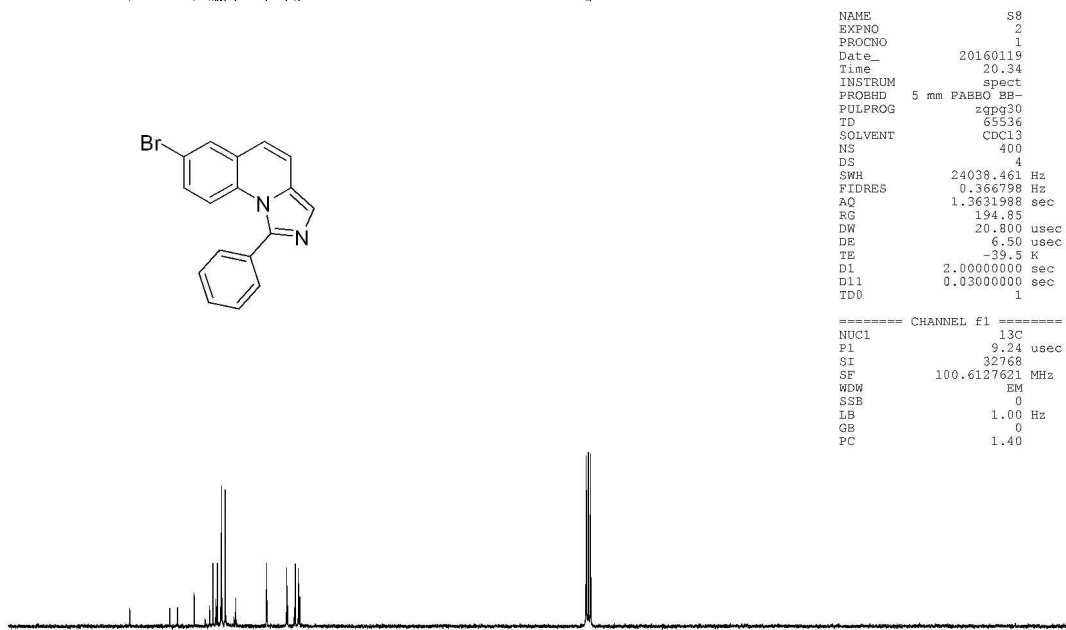

150

$\begin{array}{lll}140 & 130 \quad 120\end{array}$

$110 \quad 100$

70

60

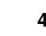

$\begin{array}{lllll}30 & 20 & 10 & 0 & \mathrm{ppm}\end{array}$ 
7-fluoro-1-phenylimidazo[1,5-a]quinoline (3ja).
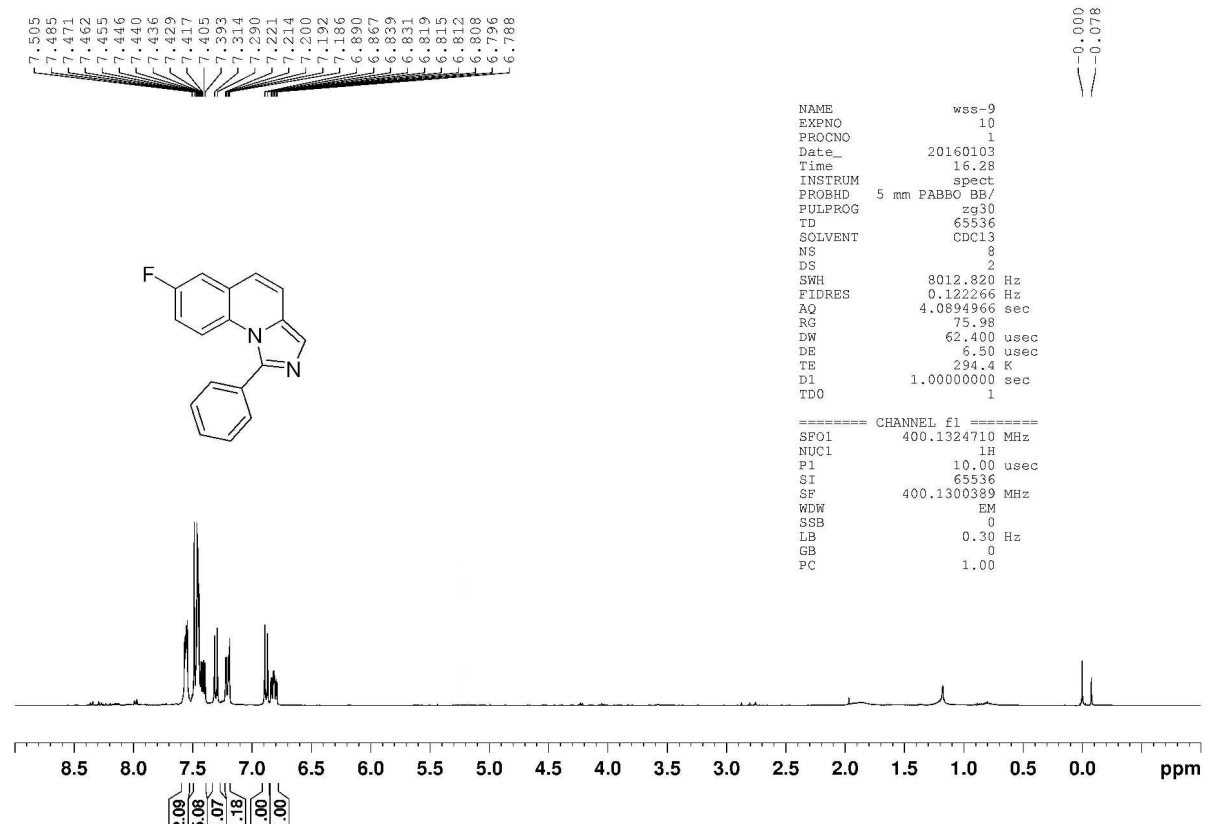
(5)

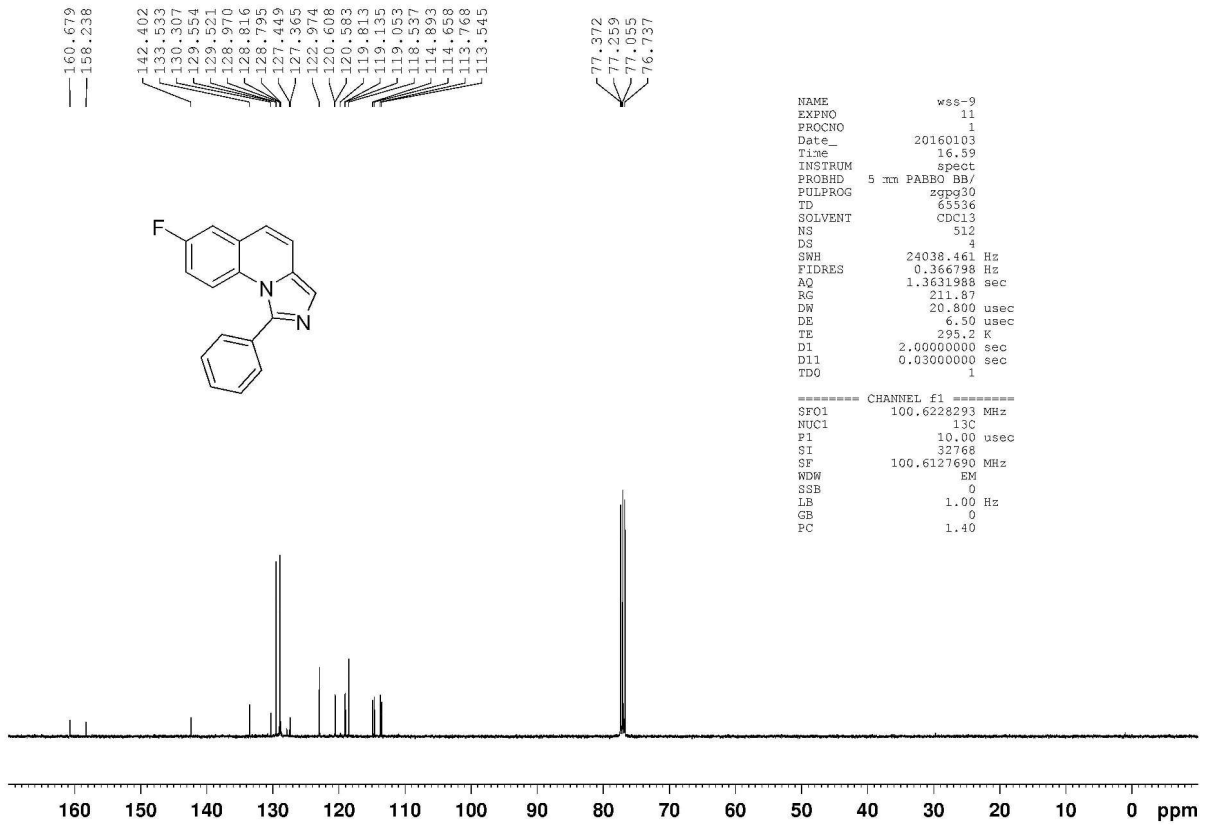


7-nitro-1-phenylimidazo[1,5-a]quinoline (3ka).
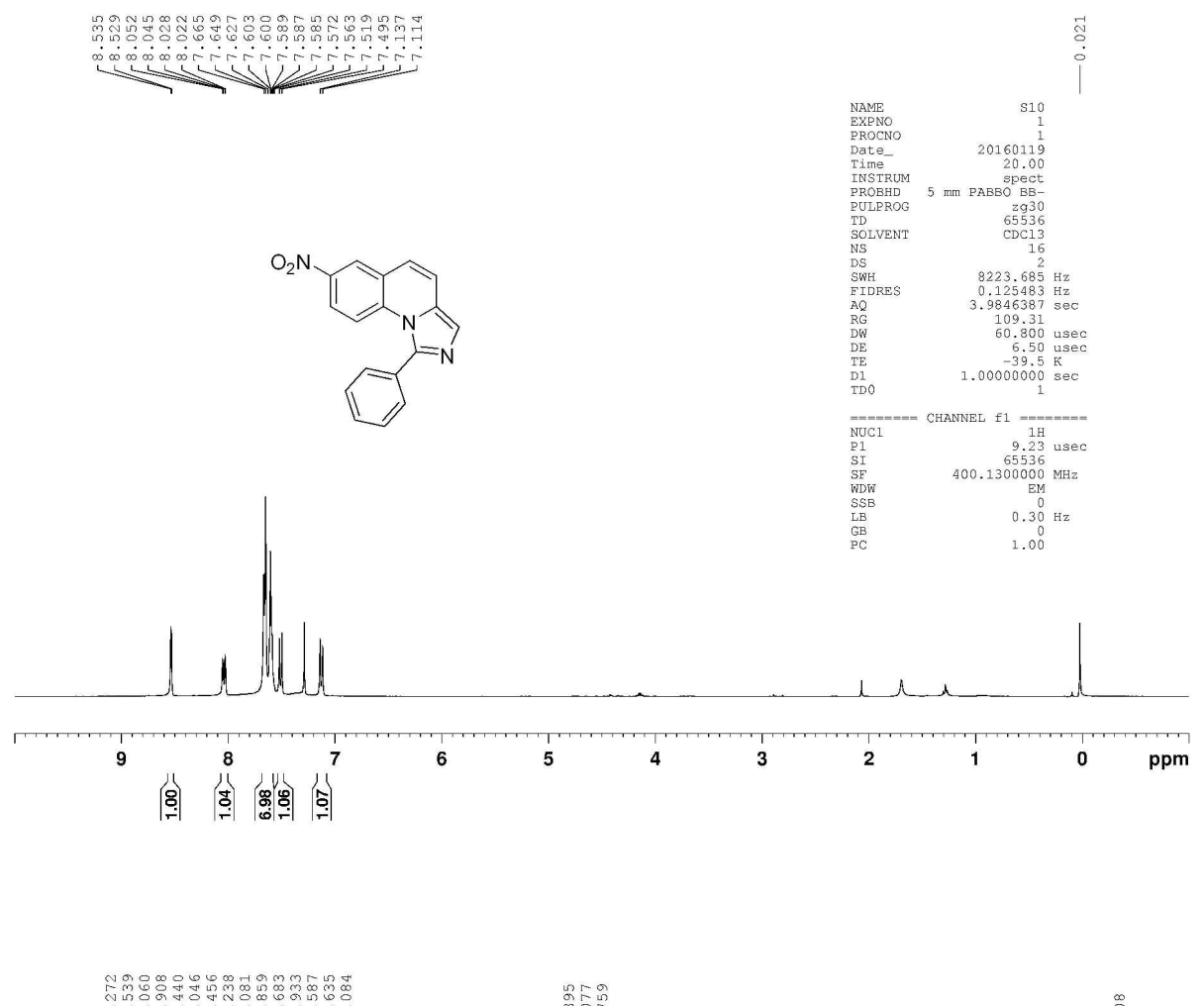

के

V V - vilil

$\sqrt{\sqrt[n]{n}}$

$\stackrel{\infty}{\circ}$
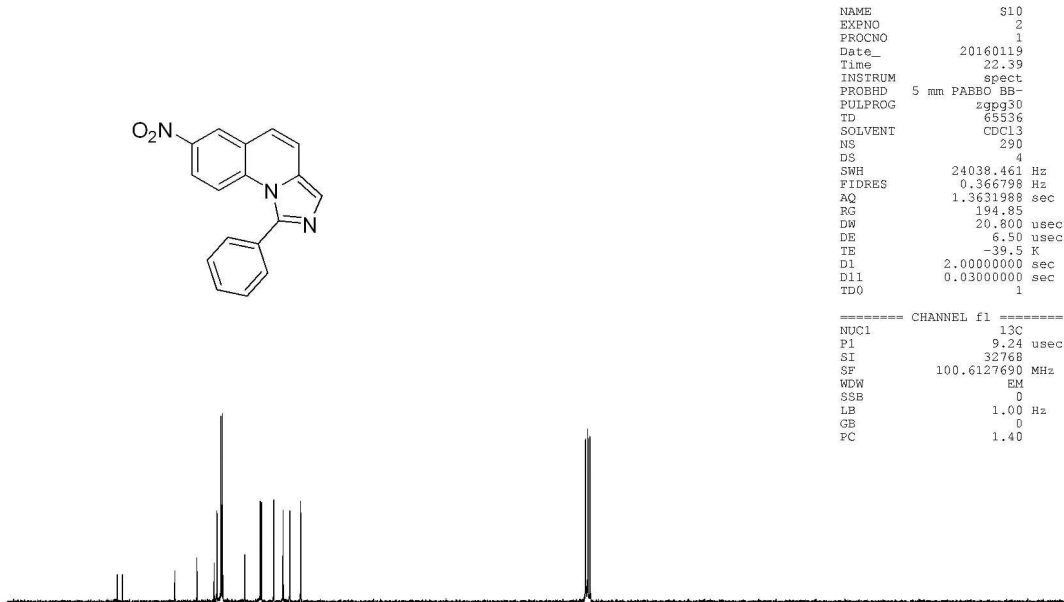

150

$\begin{array}{lllll}140 & 130 & 120 & 110 & 100\end{array}$ 
3,5-diphenylimidazo[1,5-c]quinazoline (3la).
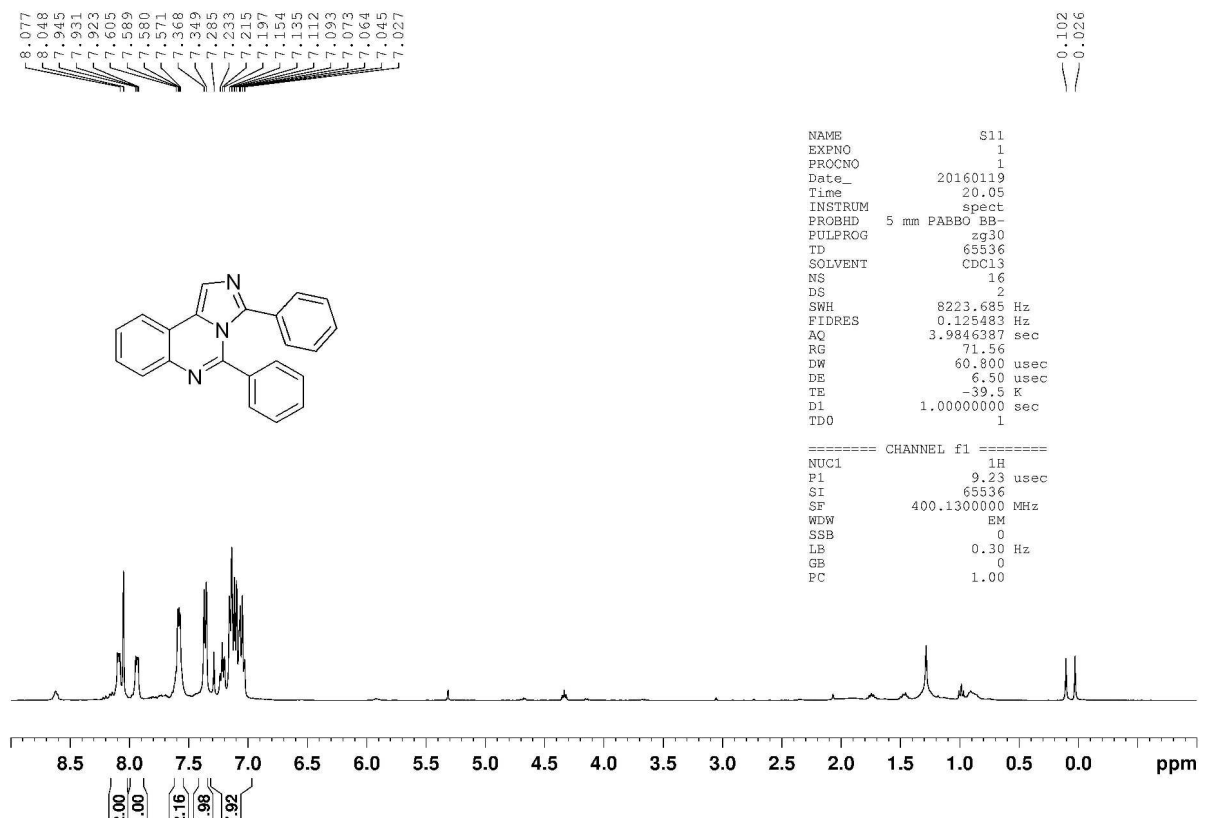
(8)

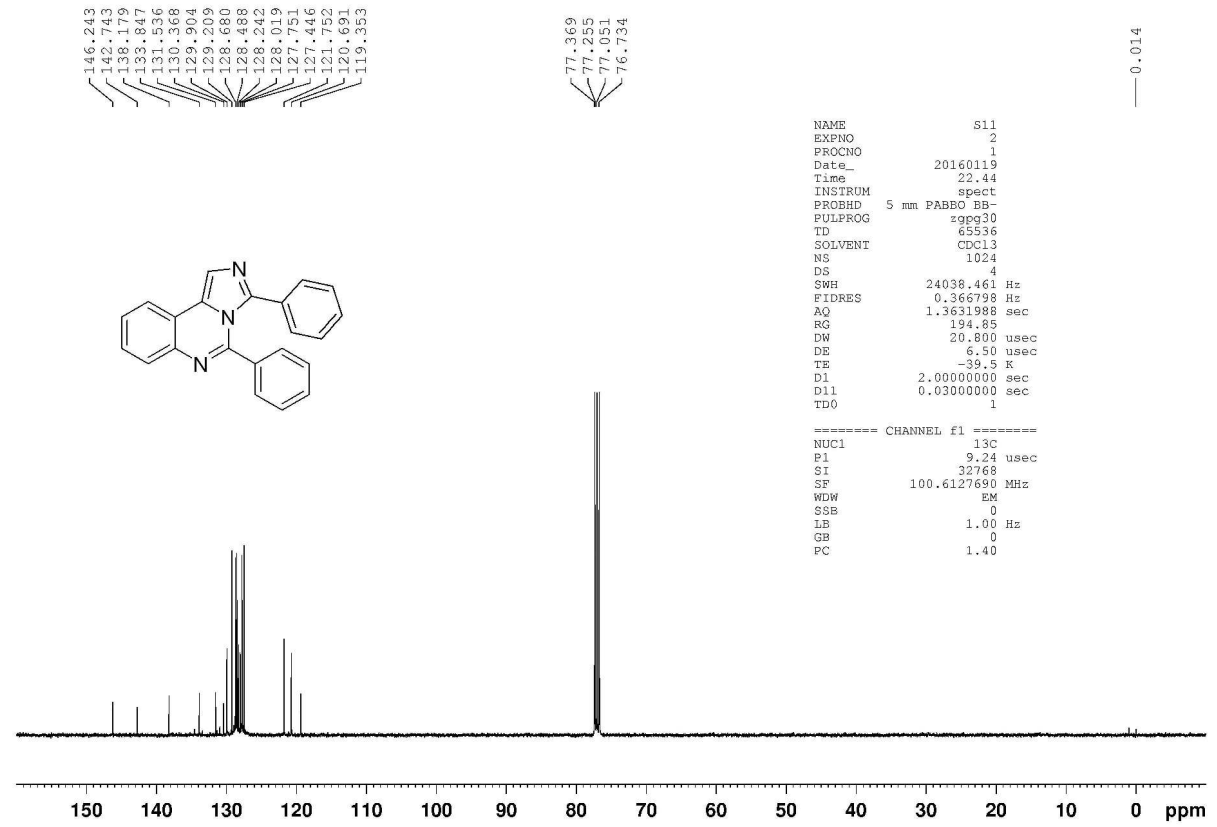

